\title{
Experimental study and DEM Modelling of Bolted Composite Lap Joints \\ Subjected to Tension
}

\author{
Min Yu ${ }^{\mathrm{a}}$, Biying Yang ${ }^{\mathrm{a}}$, Yin $\mathrm{Chi}^{\mathrm{a}}$, Jiaxin Xie ${ }^{\mathrm{a}}$, Jianqiao $\mathrm{Ye}^{\mathrm{b}, *}$ \\ a. Department of Civil Engineering, Wuhan University, Wuhan, China \\ b. Department of Engineering, Lancaster University, Lancaster LA1 4YR, UK
}

\begin{abstract}
This paper presents a numerical approach using the discrete element method to predict strength and damage propagation of plates and bolted lap joints subjected to axial tension. Tensile tests on GFRP plates and bolted joints are carried to obtained their overall stiffness and strength. A new three-dimensional discrete element model constructed by a 19-ball assembly is proposed and the relationships between the macro and the micro mechanical properties of FRP is established through calibrations using the test results. The calibrated DEM model is then used to reproduce the test results. Excellent agreements are achieved between the numerical and the experimental results in terms of not only the overall failure loads, but also the detailed failure modes, including cracking and delamination. The research shows great potential of the DEM model in predicting strength of composite materials and presenting detailed local damage and damage propagation at micro-scale, which represents a significant advantage over the conventional numerical methods, such as the finite element method.
\end{abstract}

Keywords: fiber reinforced polymer; discrete element method; strength calibration; bolted joint.

\section{Introduction}

Offering advantages over other materials in physical and mechanical properties, such as strength to weight ratio, fatigue resistance and design flexibility, fiber reinforced polymers (FRP) have been increasingly used in construction industry and many other industrial applications ${ }^{[1]}$. In civil engineering, FRP is known as an effective and economic material for strengthening and retrofitting of existing structures, and now increasingly used as load-bearing materials in structures such as bridges and buildings ${ }^{[2-4]}$. Made of fibers and polymer resins, a fiber reinforced composite is a typical kind of heterogeneous material with diverse properties ${ }^{[5]}$. A clear understanding of its failure mechanism will promote wider applications of FRP with the same confidence as with the traditional materials. Extensive researches on FRP used in structures have been carried out world-widely mainly in the last two decades, including researches on FRP ranging from materials ${ }^{[6,7]}$ to structural members ${ }^{[8,9]}$ and from FRP at room temperature ${ }^{[10,11]}$ to elevated temperature ${ }^{[12-14]}$. Theoretical, experimental and computational studies are the three main research approaches. For theoretical study, two recent World Wide Failure Exercises (WWFE-II and III) investigated a collection of representative failure cases in order to study the capabilities and uniqueness among existing popular failure criteria ${ }^{[15-17]}$. The research results have shown that some of the failure criteria 
are capable of distinguishing various failure modes. However, it is also mentioned that there is not yet solid evidence that any failure criteria was available for predicting progressive cracking or damage, and containes all what is required to support designers with a convenient and reliable tool ${ }^{[17-20]}$. Experimentally, a considerable amount of tests were conducted to study failure modes of FRP, including failure under multifarious environments, such as exposure to sulfuric acid environment ${ }^{[21]}$ and immersion in seawater ${ }^{[22]}$. Computationally, Finite Element Method(FEM) ${ }^{[23-27]}$ and Boundary Element Method(BEM) ${ }^{[28-30]}$ are generally used for accurately predicting crack initiation and stress distribution. Francesca Nerilli and Giuseppe Vairo ${ }^{[27]}$, for example, proposed a nonlinear FEM approach to predict the pin-bearing failure mechanisms for FRP bolted joints. These numerical methods have made a nice progress in predicting failure of FRP, but meanwhile they are facing challenges in simulating dynamic damage propagation and intersection due to the limitations inherited form the use of the classic continuum mechanics. The inherent multiphase structure of FRP (randomly distributed fibres, cracks, inclusions, etc.) results in complex failure/fracture of the constituent elements and their interfaces. The damage evolution and failure mechanism are complex multi-scale processes developing simultaneously from micro to macro scales, i.e., from debonding, cracking and fiber breakage to final failure. It has been widely recognized that the predictive failure models currently in use are not sufficiently accurate, especially when a FRP is under biaxial or triaxial forces, resulting in that most of FRP composite structures, e.g., bolted joints, have been conservatively designed under real loading conditions ${ }^{[18,31]}$. In order to have a better understanding of composite failure and take the full advantages offered by FRP in practical design, further investigations on the failure mechanism are demanded.

The Discrete Element Method (DEM) based on discontinuous mechanics is another method worth considering for studying composite materials. Since it was first used by Cundall and Srack ${ }^{[32,33]}$ in the study of discontinuous mechanical behavior of rock in 1971, DEM has been increasingly implemented in many other fields, such as geo-materials, granular materials, concrete, ceramics, granular-fluid and particle-gas two phase flow $^{[34]}$. DEM utilizes discrete particles that are bonded together to represent the continuity of FRP composite materials. As a particle only interacts with its neighboring particles through contacts, its dynamic responses are primarily determined by Newton's second law and force-displacement relationship ${ }^{[35,36]}$ Sheng et al. ${ }^{[37]}$ and Ismail et al. ${ }^{[38]}$ used DEM to simulate the failure process and generate the stress-strain curves of composites under transverse tensile load, which demonstrated that DEM had the advantage of tracking failure paths at microscopic scale and predicting the ultimate failure strength. Yang et al. ${ }^{[39,40]}$ studied the lateral failure and delamination of cross-laminated composites and predicted damage density by two-dimensional discrete element method. Maheo et al. ${ }^{[41]}$ simulated the failure of composites under uniaxial tensile loading. Le et al. ${ }^{[42]}$ simulated the failure of FRP laminates such as fiber degumming, delamination, matrix cracking and fiber breakage. These existing studies showed that DEM is able to effectively determine initial crack and fiber/matrix cracking distribution with microscopic modeling approaches ${ }^{[35,36]}$.

To the authors' best knowledge, current researches on DEM composite modelling is predominately at micro or meso scale, such as study in fiber/matrix debonding ${ }^{[41]}$, and these studies have achieved great success. At a larger scale, Zha, et $\mathrm{a}^{[34]}$ used DEM to simulate failure of metal skinned sandwich panels subjected to uniform pressure and this is one of few articles on simulating large-scale members. However, the work did not provide the information on how they calibrated the micromechanical parameters of the model, a process that is essential 
and challenging when applying DEM to industrial problems ${ }^{[43]}$, especially when the materials are anisotropic, such as the FRP investigated in this paper.

This paper aims to propose a simplified 3D DEM model to simulate laminated plates and bolted joints. In Section 2, a 19-ball 3D unit of DEM model is proposed and equations are derived for calculating the microscopic stiffness constants of the FRP laminates material by considering each lamina as transversely isotropic. Experimental tests of GFPR plates are carried out in Section 3 to calibrate the micro contact strength through comparisons with the macro properties of the materials, such as the global strength, damage initiation and propagation. In Section 4, bolted GFRP joints with different lap length under tension are tested, and DEM models of the joints are developed. Comparisons are made to validate the applicability and accuracy of the method. Finally, conclusions and recommendations are drawn in Section 5.

\section{Three-dimensional DEM model}

\subsection{Discrete element method and basic formulation}

The Discrete element method (DEM) divides an elastic solid structure into many rigid or deformable discrete particles linked by different contacts with, normally, rectangular, hexagonal or random arrangement. In this paper, the discrete element commercial software PFC3D5.0 developed by ITASCA ${ }^{[44]}$ is used as the platform to simulate 3D composite materials, where the linear parallel-bond model is adopted to represent the interactions between the particles. The linear parallel bond can be envisioned by a set of independent elastic springs with normal and tangential stiffness, uniformly distributed over either a rectangular (in 2D) or circular (in 3D) crosssection on the contact plane or centered at the contact point as shown in Fig. 1(a $)^{[44]}$. The linear parallel bond includes two interfaces: an infinitesimal, linear elastic (no tension) and frictional interface that carries forces and a finite-size, linear elastic and bonded interface that carries forces and moments. For the liner bond, $k_{n}$ and $k_{\mathrm{s}}$ are normal and tangential stiffness, respectively; $g_{s}$ is surface gap and $\mu$ is friction coefficient. For the parallel-bond group, $\bar{k}_{n}$ and $\bar{k}_{\mathrm{s}}$ are normal and tangential stiffness, respectively. In Fig. 1(b), $\bar{\sigma}_{c}$ denotes tensile strength and $\bar{c}$ and $\bar{\Phi}$ are the cohesion and friction angles, respectively, which are related to shear strength $\bar{\sigma}_{s}$ (when $\bar{c}=0, \bar{\sigma}_{s}=\bar{c}$ ). The parallel bond resists also relative rotation, and is linear elastic until the strength limit is exceeded and the bond breaks. In this paper, the parallel bond is used to simulate the strength of the contacts. Thus, to define a linear parallel bond, four parameters are required: i.e., the normal stiffness $\bar{k}_{n}$, the tangential stiffness $\bar{k}_{s}$, the normal strength $\bar{\sigma}_{n}$ and the tangential strength $\bar{\sigma}_{s}$. The determination of these parameters is a key step of DEM simulation, which directly affects the accuracy and efficiency of the model. In principle, a theoretical relationship can be established between the bond stiffness (i.e. the micro stiffness) and the material's global elastic stiffness (i.e. the macro stiffness).

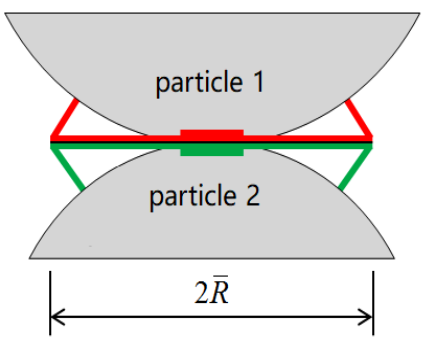

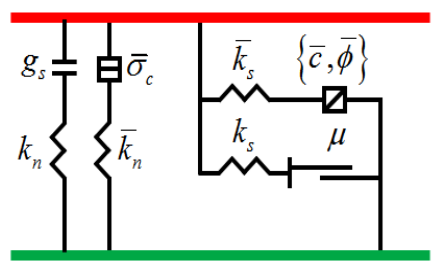

bonded 

(a) contact between two particle
(b) mechanism for linear parallel bond

Fig. 1 Linear parallel bond model

In a DEM model, the deformation of a material is simulated by the deformation of the springs. The formulas for calculating the spring constants of isotropic materials were derived by some researchers, such as Taverez and Plesha ${ }^{[45]}$. Liu and Liu ${ }^{[46]}$ proposed a general formula for both anisotropic and isotropic materials in 2D. Liu et al. ${ }^{[47]}$ and Zhao et al. ${ }^{[48]}$ also developed an average strain energy method to correlate bond stiffness with 3D macro material properties. The average strain energy was calculated on the basis of relative displacement of the particles. Based on Liu and $\mathrm{Liu}^{[46]}$, the three dimensional average strain energy can be derived as below.

Considering that particle $i$ is connected to $p$ neighboring particles that are numbered $j(j=1,2, \ldots, p)$, the average strain energy density $U_{i}$ around particle $i$ is written as follows:

$$
U_{i}=\frac{1}{V_{\mathrm{i}}} \sum_{j=1}^{p} \frac{1}{2}\left[\frac{1}{2} k_{n i j}\left(u_{n j}-u_{n i}\right)^{2}+\frac{1}{2} k_{s i j}\left(u_{s j}-u_{s i}\right)^{2}\right]
$$

where $V_{\mathrm{i}}$ is the volume of the outside cube of the inscribed particle $i ; k_{n i j}$ and $k_{\mathrm{s} i j}$ are the spring constants between particles $i$ and $j$ in the normal and tangential directions, respectively; $u_{n i}$ and $u_{s i}$ are the normal and tangential displacements of particle $i ; u_{n j}$ and $u_{s j}$ are the normal and the tangential displacements of particle $j$.

Assuming that the directional cosines of the normal of the contact between particles $i$ and $j$ is $(l, m$, $n)$, one has

$$
\left\{\begin{array}{l}
\left(u_{n j}-u_{n i}\right)^{2}=\left[\left(u_{x j}-u_{x i}\right) l+\left(u_{y j}-u_{y i}\right) m+\left(u_{z j}-u_{z i}\right) n\right]^{2} \\
\left(u_{s j}-u_{s i}\right)^{2}=\left[\left(u_{x j}-u_{x i}\right)^{2}+\left(u_{y j}-u_{y i}\right)^{2}+\left(u_{z j}-u_{z i}\right)^{2}\right]-\left(u_{n j}-u_{n i}\right)^{2}
\end{array}\right.
$$

Considering the displacement-strain relationship, the following equations are obtained.

$$
\left\{\begin{array}{l}
u_{x j}-u_{x i}=\left(x_{j}-x_{i}\right) \varepsilon_{x}+\frac{y_{j}-y_{i}}{2} \gamma_{x y}+\frac{z_{j}-z_{i}}{2} \gamma_{z x}=D_{i j} l \varepsilon_{x}+\frac{D_{i j} m}{2} \gamma_{x y}+\frac{D_{i j} n}{2} \gamma_{z x} \\
u_{y j}-u_{y i}=\frac{x_{j}-x_{i}}{2} \gamma_{x y}+\left(y_{j}-y_{i}\right) \varepsilon_{y}+\frac{z_{j}-z_{i}}{2} \gamma_{y z}=\frac{D_{i j} l}{2} \gamma_{x y}+D_{i j} m \varepsilon_{y}+\frac{D_{i j} n}{2} \gamma_{y z} \\
u_{z j}-u_{z i}=\frac{x_{j}-x_{i}}{2} \gamma_{z x}+\frac{y_{j}-y_{i}}{2} \gamma_{y z}+\left(z_{j}-z_{i}\right) \varepsilon_{z}=\frac{D_{i j} l}{2} \gamma_{z x}+\frac{D_{i j} m}{2} \gamma_{y z}+D_{i j} n \varepsilon_{z}
\end{array}\right.
$$

where $\left(x_{i}, y_{i}, z_{i}\right)$ and $\left(x_{j}, y_{j}, z_{j}\right)$ are, respectively, the coordinates of particles $i$ and $j ; D_{i j}$ is the distance between the center of particles $i$ and $j$.

\subsection{Relationships between spring constants and the elastic constants of material}

Eq.(1-3) are the three basic equations that apply to any material and any form of particle arrangement. Lei et al. ${ }^{[36]}$ compared and evaluated three different particle packings, namely, the $3 \mathrm{D}$ discrete Lattice model, the 3D hexagonal close packing model and the extended 2D hexagonal and square packing model. It was found that the extended 2D hexagonal and square model is more suitable and efficient for modelling composite lamina. In this 
paper, on the basis of the nine-disc model proposed by Liu ${ }^{[46]}$, which is similar to the extended 2D square model mentioned above, a new 9-ball model, as shown in Fig. 2, is proposed. The new 19-ball model is a reduction of the 27-ball cube shown in Fig. 3 by removing the connections between the central ball and the balls at the eight corners (vertices).

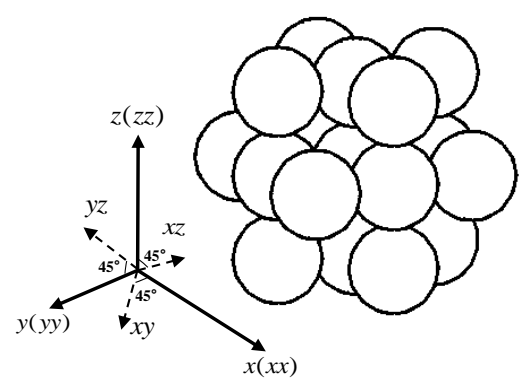

Fig. 2 19-ball modelling unit

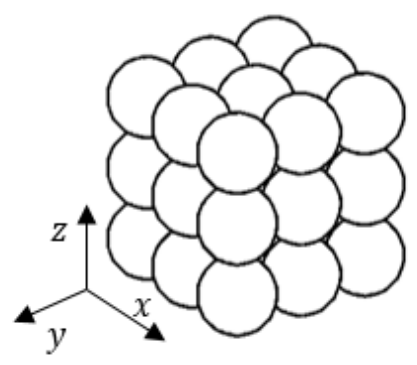

Fig. 3 27-ball cube

Six springs, respectively, along the $x(x x), y(y y), z(z z), x y, x z$ and $y z$ directions are introduced to represent the normal and tangent contact stiffness. The distances between the central ball $i$ and the rest 18 balls are either $2 R$ or $2 \sqrt{2} R$, where $R$ is the particle radius. The average strain energy density of the central ball of Fig. 2 can be expressed in terms of the spring constants and the strains below by following Eq.(1)-(3).

$$
U=\frac{R}{2 V}\left[\begin{array}{l}
\left(2 k_{n x x}+2 k_{n x y}+2 k_{n x z}+2 k_{s x y}+2 k_{s x z}\right) \varepsilon_{x}^{2}+\left(4 k_{n x y}-4 k_{s x y}\right) \varepsilon_{x} \varepsilon_{y}+\left(4 k_{n x z}-4 k_{s x z}\right) \varepsilon_{x} \varepsilon_{z}+ \\
\left(2 k_{n x y}+2 k_{n y y}+2 k_{n y z}+2 k_{s x y}+2 k_{s y z}\right) \varepsilon_{y}^{2}+\left(4 k_{n y z}-4 k_{s y z}\right) \varepsilon_{y} \varepsilon_{z}+ \\
\left(2 k_{n x z}+2 k_{n y z}+2 k_{n z z}+2 k_{s x z}+2 k_{s y z}\right) \varepsilon_{z}^{2}+\left(2 k_{n y z}+k_{s x y}+k_{s x z}+\frac{k_{s y y}}{2}+\frac{k_{s z z}}{2}\right) \gamma_{y z}^{2}+ \\
\left(2 k_{n x z}+k_{s x y}+k_{s y z}+\frac{k_{s x x}}{2}+\frac{k_{s z z}}{2}\right) \gamma_{x z}^{2}+\left(2 k_{n x y}+k_{s x z}+k_{s y z}+\frac{k_{s x x}}{2}+\frac{k_{s y y}}{2}\right) \gamma_{x y}^{2}
\end{array}\right]
$$

where $U$ is the average strain energy density. $V$ is the volume of the representative cuboid occupied by a ball, which is $(2 R)^{3} . k_{n i j}$ and $k_{\mathrm{s} i j}$ are, respectively, the six normal and the six tangential spring constants relative to the respective directions shown in Fig. 2. $\varepsilon_{x}, \varepsilon_{y}, \varepsilon_{z}, \gamma_{x y}, \gamma_{y z}$ and $\gamma_{z x}$ are the engineering strain components.

From the theory of three-dimensional elasticity, the average strain energy density ${ }^{[1]}$ of an anisotropic material is written as

$$
U=\left\{\begin{array}{l}
\frac{1}{2} c_{11} \varepsilon_{x}^{2}+c_{12} \varepsilon_{x} \varepsilon_{y}+c_{13} \varepsilon_{x} \varepsilon_{z}+c_{14} \varepsilon_{x} \gamma_{x y}+c_{15} \varepsilon_{x} \gamma_{z x}+c_{16} \varepsilon_{x} \gamma_{x y} \\
+\frac{1}{2} c_{22} \varepsilon_{y}^{2}+c_{23} \varepsilon_{y} \varepsilon_{z}+c_{24} \varepsilon_{y} \gamma_{x y}+c_{25} \varepsilon_{y} \gamma_{z x}+c_{26} \varepsilon_{y} \gamma_{x y} \\
+\frac{1}{2} c_{33} \varepsilon_{z}^{2}+c_{34} \varepsilon_{z} \gamma_{x y}+c_{35} \varepsilon_{z} \gamma_{z x}+c_{36} \varepsilon_{z} \gamma_{x y} \\
+\frac{1}{2} c_{44} \gamma_{x y}^{2}+c_{45} \gamma_{x y} \gamma_{z x}+c_{46} \gamma_{x y} \gamma_{x y} \\
+\frac{1}{2} c_{55} \gamma_{x y}^{2}+c_{56} \gamma_{z x} \gamma_{x y} \\
+\frac{1}{2} c_{66} \gamma_{x y}^{2}
\end{array}\right\}
$$


where $c_{11}, c_{12}, \ldots, c_{66}$ are elastic coefficients of the material; The subscripts, 1,2 and 3 , are related to the $x$, $y$ and $z$ directions, respectively.

Since the average strain energy density in Eq.(4) and (5) must be equal, the following equations are obtained for an orthotropic material

$$
\left\{\begin{array}{l}
k_{n x x}+k_{n x y}+k_{n x z}+k_{s x y}+k_{s x z}=\delta c_{11} \\
k_{n x y}+k_{n y y}+k_{n y z}+k_{s x y}+k_{s y z}=\delta c_{22} \\
k_{n x z}+k_{n y z}+k_{n z z}+k_{s x z}+k_{s y z}=\delta c_{33} \\
k_{n x y}-k_{s x y}=\delta c_{12} \\
k_{n y z}-k_{s y z}=\delta c_{23} \\
k_{n x z}-k_{s x z}=\delta c_{13} \\
4 k_{n y z}+2 k_{s x y}+2 k_{s x z}+k_{s y y}+k_{s z z}=4 \delta c_{44} \\
4 k_{n x z}+2 k_{s x y}+2 k_{s y z}+k_{s x x}+k_{s z z}=4 \delta c_{55} \\
4 k_{n x y}+2 k_{s x z}+2 k_{s y z}+k_{s x x}+k_{s y y}=4 \delta c_{66}
\end{array}\right.
$$

where $\delta=V /\left(4 R^{2}\right)=2 R$.

Eq.(6) shows the relationship between the spring constants and the macro elastic constants for the 19-ball model of general orthotropic materials. As a part of validations, Eq.(6) is reduced to a two-dimensional case that has been investigated by Liu and Liu ${ }^{[46]}$. In a two-dimensional case, all the constants that are relative to the outof-plane direction, e.g., the $z$ direction, are ignored. Thus Eq.(6) is reduced to

$$
\left\{\begin{array}{l}
k_{n x x}+k_{n x y}+k_{s x y}=\delta c_{11} \\
k_{n x y}+k_{n y y}+k_{s x y}=\delta c_{22} \\
k_{n x y}-k_{s x y}=\delta c_{12} \\
4 k_{n x y}+k_{s x x}+k_{s y y}=4 \delta c_{66}
\end{array}\right.
$$

Letting $k_{n x x}=k_{n 1}, k_{n y y}=k_{n 2}, k_{n x y}=k_{n 3}$, and $k_{s x x}=k_{s y y}=k_{s x y}=k_{s}$, it can be shown that the solutions of Eq.(7) are identical to the solutions of Eq.(19) in Liu and $\mathrm{Liu}^{[46]}$ for a $2 \mathrm{D}$ orthotropic material.

When Eq.(6) is applied to general orthotropic materials, the number of the unknown contact stiffness is greater than that of equations. Reasonable assumptions can be made at this stage to solve the problem. For a thin plate under tension, where the $z$ axis is assumed in the thickness direction, Eq.(6) can be simplified by ignoring two pairs of contacts, i.e., $k_{n x z}, k_{s x z}$ and $k_{n y z}, k_{s y z}$ in the 19-ball model, as shown in Fig. 4, where only the contacts between the balls are shown. In this circumstance, $\mu_{13}, \mu_{31}, \mu_{23}, \mu_{32}$ are ignored.
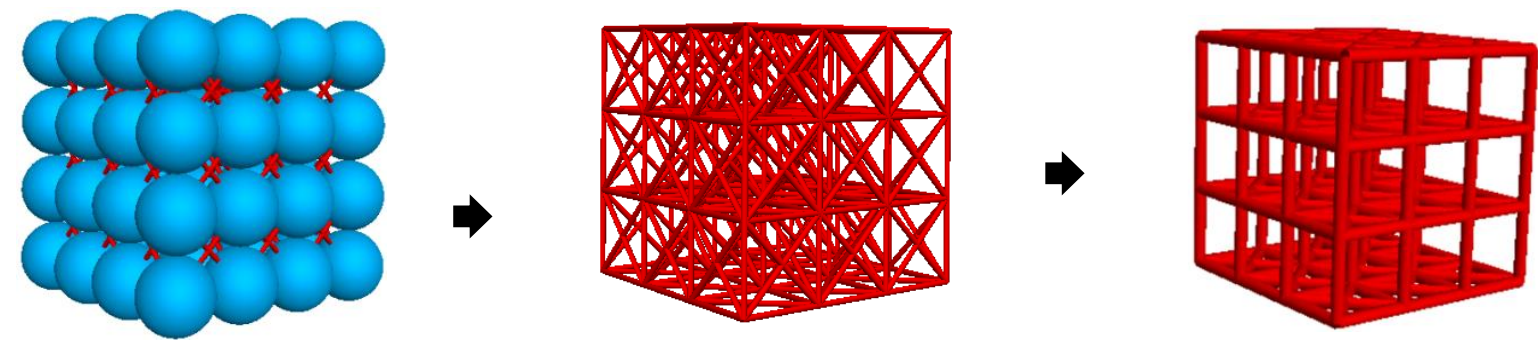

Fig. 4 model showing contacts

Assuming that $k_{s x x}=k_{s y y}=k_{s x y}$, the normal and the tangential spring constants in Eq.(6) are obtained as 


$$
\left\{\begin{array}{l}
k_{n x x}=\frac{\delta}{3}\left(3 c_{11}+c_{12}-4 c_{66}\right) \\
k_{n y y}=\frac{\delta}{3}\left(c_{12}+3 c_{22}-4 c_{66}\right) \\
k_{n x y}=\frac{\delta}{3}\left(c_{12}+2 c_{66}\right) \\
k_{s x x}=k_{s y y}=k_{s x y}=\frac{2 \delta}{3}\left(c_{66}-c_{12}\right) \\
k_{n z z}=\delta c_{33} \\
k_{s z z}=2 \delta\left(c_{12}+2 c_{44}-c_{66}\right)
\end{array}\right.
$$

where $\delta=V /\left(4 R^{2}\right)=2 R, \quad c_{11}=1 /\left(E_{2} E_{3} \Delta\right), \quad c_{12}=\mu_{21} /\left(E_{1} E_{3} \Delta\right), \quad c_{22}=1 /\left(E_{1} E_{3} \Delta\right), \quad c_{33}=\left(1-\mu_{12} \mu_{21}\right) /\left(E_{1} E_{2} \Delta\right)$, $c_{44}=G_{23}, \quad c_{66}=G_{12}$ and $\Delta=\left(1-\mu_{12} \mu_{21}\right) /\left(E_{1} E_{2} E_{3}\right)$. It is shown that the contact stiffness has linear relationship with the size of the particles. It is worthy of noting that the derived formulas for calculating the contact stiffness between particles are not restricted to the material studied in this paper, so they can be used for any material as long as the 19 ball unit is used in the model.

\section{DEM model for FRP plates}

\subsection{Experimental tests on GFRP laminates}

In order to calibrate the micro parameters of the laminated plates for the DEM model, laboratory tensile tests were carried out on GFRP laminates. The laminates used in this study are EXTREN 500 Series pultruded GFRP manufactured by Strongwell. In nature, the material of 500 Series is constructed by glass fibres and polyester resin matrix whose weight percentage are respectively about $30 \%$ and $70 \%$. The glass fibre reinforcement has two forms, including rovings and continuous filament mat (CFM). The rovings are unidirectional parallel fibre bundles and mainly determine the longitudinal strength and stiffness of the plates, while the CFM contributes to these properties in the transverse direction and is usually placed within the stacking sequence ${ }^{[49]}$. Resin matrix includes $10 \%$ of calcium carbonate or kaolin filler. The plate is a laminate of five identical layers. Specimens with and without hole were cut from the composite for testing. The longitudinal cuts of the specimens without holes (Fig.5a) are either parallel or perpendicular to the roving direction, so that their elastic modules and ultimate strengths in the two directions can be determined, respectively. Plates with holes with longitudinal cuts in the roving diretion is desined as shown in Fig.5(b) to figure out shear properites in the plane.

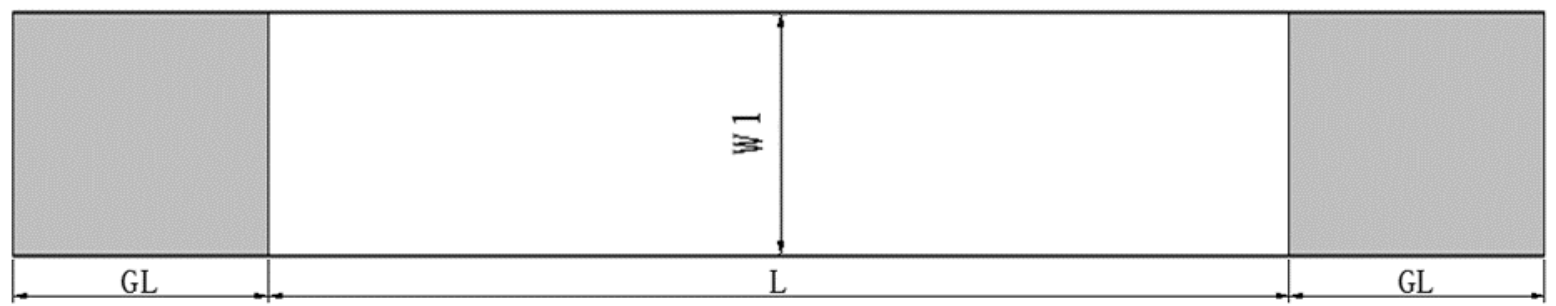

(a) GFRP plate without hole 


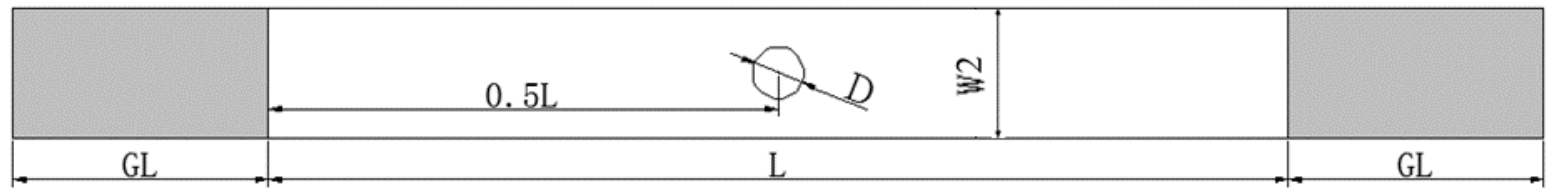

(b) GFRP plate with a hole

Specimen length: $\mathrm{L}=200 \mathrm{~mm} \quad$ Specimen width: $\mathrm{W} 1=40 \mathrm{~mm} \quad$ Specimen width: $\mathrm{W} 2=25 \mathrm{~mm}$

Specimen thickness: $\mathrm{H}=6.4 \mathrm{~mm} \quad$ Hole diameter: $\mathrm{D}=10 \mathrm{~mm} \quad$ Grip length: $\mathrm{GL}=50 \mathrm{~mm}$

Fig. 5 Details of GFRP specimens used in the tension plate tests

The tensile tests of the GFRP laminates were completed on a $300 \mathrm{kN}$ universal testing machine under displacement control at a rate of $1 \mathrm{~mm} / \mathrm{min}$. In order to accurately measure the deformation of the specimens, especially of the joints tested later, the IMETRUM non-contact video gauge, as shown in Fig. 6, was used to capture high resolution images of the dynamic deformation process. The basic principle of the non-contact video gauge is utilizing the image speckle recognition and the two-dimensional image processing techniques to accurately identify and track the deformation of the surface of the object under test, and accurately measure the relative displacement of the target points, as shown by Fig. 7 .

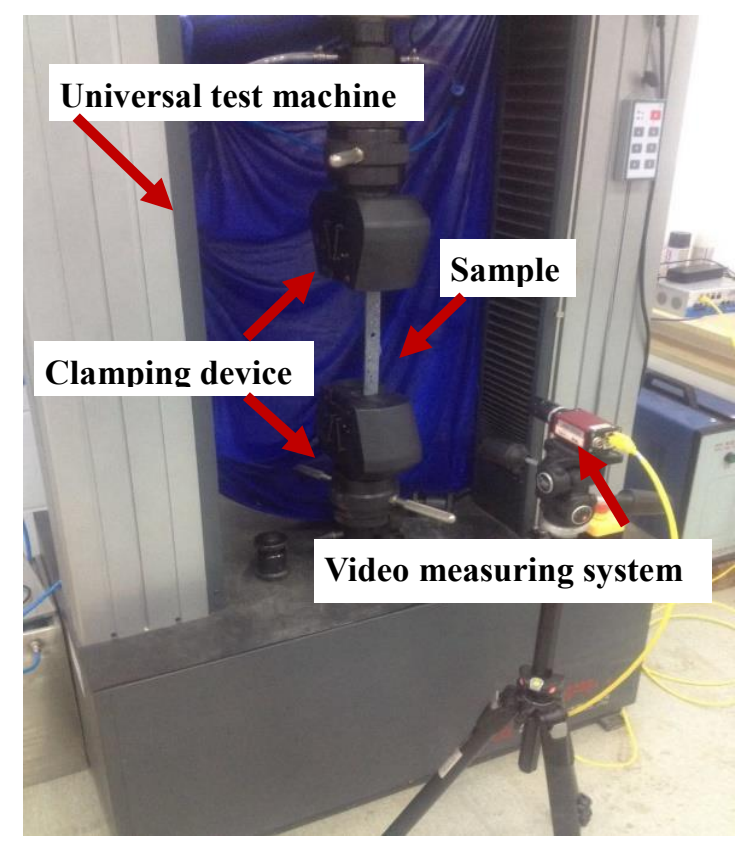

Fig. 6 Test equipments

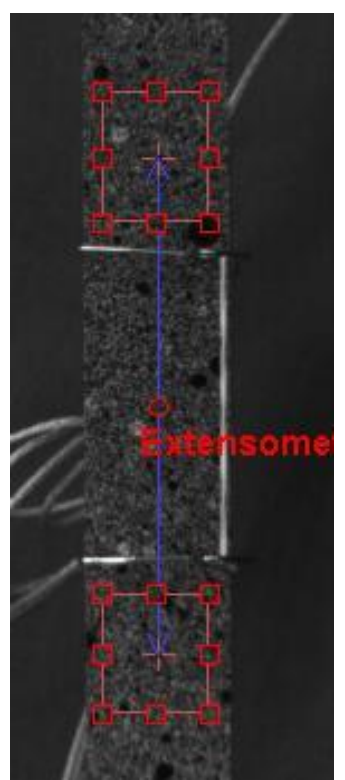

Fig. 7 Displacement tracking

Two groups of plates, i.e., with and without central holes, were tested for stiffness, strength and damage propagation. In each group, there were 3 nominally identical laminates. In the tensile tests of the plates without holes, the development of the transverse cracks caused fracture of the outer layer and then final brittle failure of the laminate as shown in Error! Reference source not found. (a). The failure mode of the plates with holes in Error! Reference source not found.(b) shows transverse cracks across the central hole, where the crosssection is weakened. The transverse cracks propagated quickly, and a total failure occurred causing dramatic reduction of the bearing capacity. Since the holes were not exactly centralized resulting in slightly unsymmetrical deformation. 


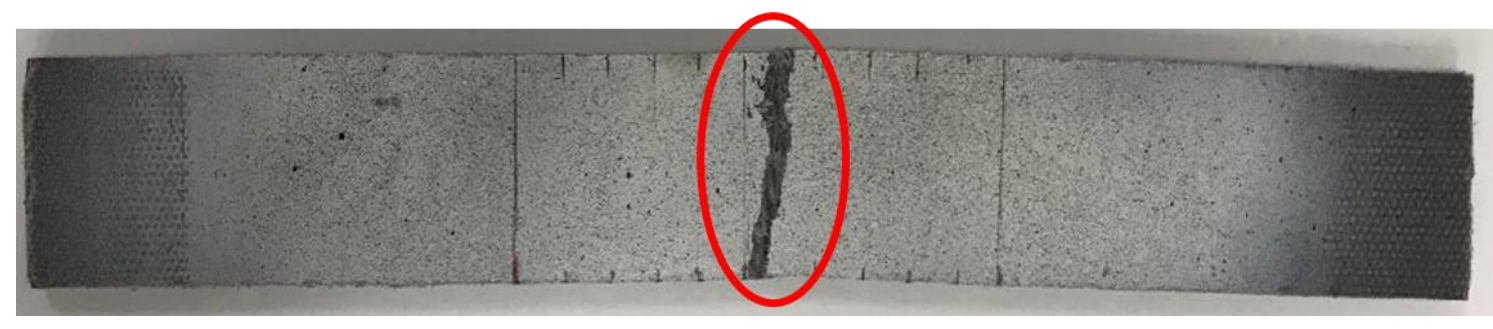

(a) without hole

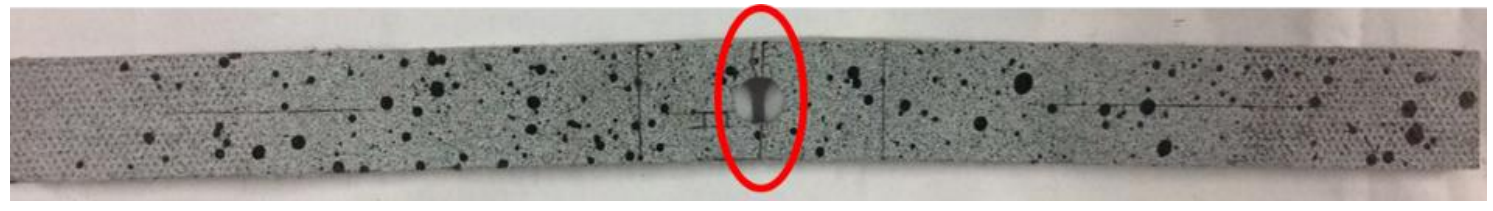

(b) with hole

Fig. 8 Failure modes of plate with and without hole

The test results are shown in Table 1 where the data provided by the Manufacture and the test results of Turvey and Szulik ${ }^{[49]}$ are also presented. Comparing to the data provided by the manufacturer and the same group plates tested by Turvey and Szulik ${ }^{[49]}$. It can be seen from the table that some of the test results from both this research and Turvey and Szulik ${ }^{[49]}$ 's work are significantly different to the ones from the manufacturer datasheet that represent the minimum property values of the material. Nevertheless, we believe that our test results are reasonable representations of typical properties of the material and use them to calibrate the micro contact properties for the DEM model.

Table 1 Test results and comparison

\begin{tabular}{llllll}
\hline & & Test & Manufacturer & Turvey and Szulik \\
\hline \multirow{4}{*}{$\begin{array}{l}\text { Plates } \\
\text { holes }\end{array}$} & Elasticity modulus $E_{1}$ & $17.46 \mathrm{GPa}$ & $17.20 \mathrm{GPa}$ & $23.13 \mathrm{GPa}$ \\
\cline { 2 - 6 } & Elasticity modulus $E_{2}$ & $5.52 \mathrm{GPa}$ & $5.52 \mathrm{GPa}$ & - \\
\cline { 2 - 6 } & Shear modulus $G_{12}$ & - & $2.93 \mathrm{GPa}$ & - \\
\cline { 2 - 6 } & Poisson's ratio $\mu_{21}$ & $0.303 \mathrm{~mm} / \mathrm{mm}$ & $0.330 \mathrm{~mm} / \mathrm{mm}$ & - \\
\cline { 2 - 6 } & Longitudinal strength $\sigma_{x}$ & $293.0 \mathrm{MPa}$ & $207.0 \mathrm{MPa}$ & $300.0 \mathrm{MPa}$ \\
\cline { 2 - 5 } & Transverse strength $\sigma_{y}$ & $95.4 \mathrm{MPa}$ & $48.3 \mathrm{MPa}$ & - \\
\hline Plates with holes & Tension capacity & $21.6 \mathrm{kN}$ & - & - \\
\hline
\end{tabular}

\subsection{DEM model of GFRP laminates}

Instead of simulating individual fibres within the matrix of each layer, which is practically possible if the distribution of the fibres are known, though it will be more computational intensive, each of the FRP layer is considered as a homogenous and transversely isotropic material that are bonded together to form the laminate. Initial tests on the size of particle were carried out, which shown that by dividing the plates into 4-6 layers (4-6 particles across the thickness), satisfactory results can be achieved without significantly increase the computational costs. It was decided to use 5 layers in the model, which was also because, by inspection, the tested laminates were made of 5 identical laminas. For the model of the plates with hole, the diameter of the hole is $10 \mathrm{~mm}$, the same as that of tested specimens. These models are shown in Fig.9 Error! Reference source not found.(a), where the longitudinal, the width and the thickness directions are defined as the $x$-, $y$ - and $z$-directions, respectively. 
On the basis of the simplified 19-ball model in Section 2.2, there are four normal and four tangential contact stiffness that can be calculated from Eq.(8) using the tested elastic constants presented in Table 1 and assuming that $E_{3}=E_{2}, G_{23}=G_{12}$ for the transversely isotropic material. The calculated stiffness for the balls within each of the lamina are summarized below in Table 2 .

Table 2 contact stiffness

\begin{tabular}{cllll}
\hline $\begin{array}{c}\text { Normal stiffness } \\
\mathrm{MPa} / \mathrm{m}\end{array}$ & $k_{n x x}$ & $k_{n y y}$ & $k_{n x y}$ & $k_{n z z}$ \\
\cline { 2 - 5 } & $18.318 \times 10^{6}$ & $2.941 \times 10^{6}$ & $3.159 \times 10^{6}$ & $6.900 \times 10^{6}$ \\
\hline Tangential stiffness & $k_{s x x}$ & $k_{s y y}$ & $k_{s x y}$ & $k_{s z z}$ \\
\cline { 2 - 5 } $\mathrm{MPa} / \mathrm{m}$ & $1.006 \times 10^{6}$ & $1.006 \times 10^{6}$ & $1.006 \times 10^{6}$ & $11.631 \times 10^{6}$ \\
\hline
\end{tabular}

On the contacts, there are also four normal strength, i.e., $\bar{\sigma}_{n x x}, \bar{\sigma}_{n x y}, \bar{\sigma}_{n y y}, \bar{\sigma}_{n z z}$, and four tangential strength, i.e., $\bar{\sigma}_{s x x}, \bar{\sigma}_{s x y}, \bar{\sigma}_{s y y}$ and $\bar{\sigma}_{s z z}$ required to be determined.
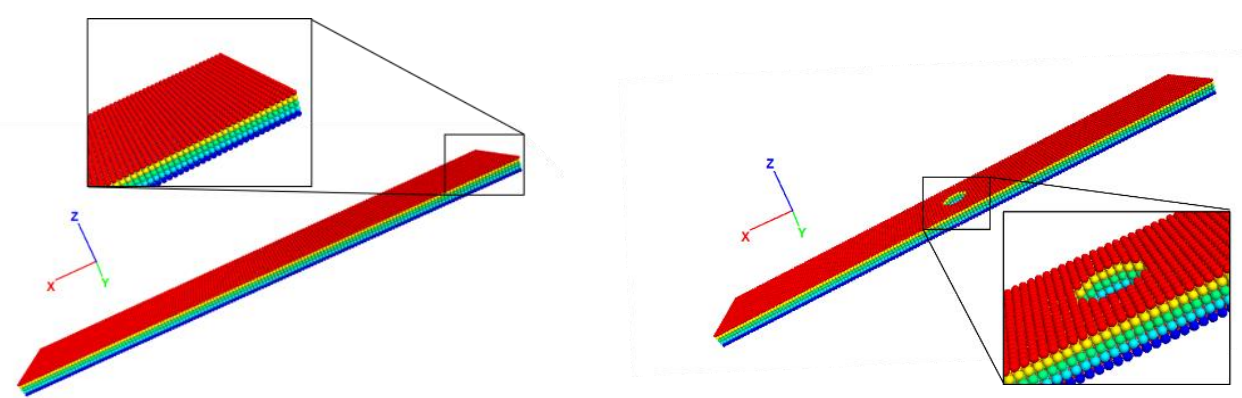

(a) five-layer model
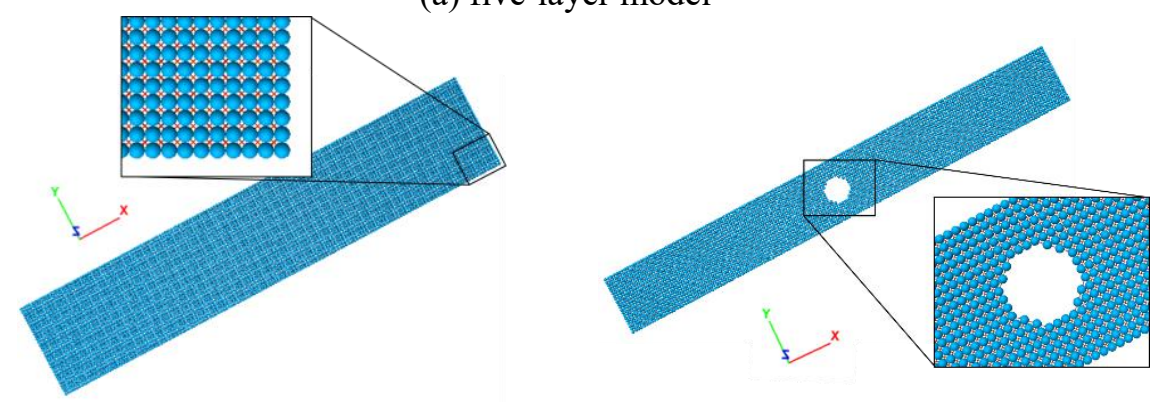

(b) single layer model

Fig. 9 3D model of laminates

For the thin plate subjected to uniaxial tension in the fibre direction, numerical simulations have been carried out for the multi-layer models to calibrate the strength of the bonds in the x-y plane. Through parametric analysis of this model, it is found that tangential strength plays a negligible role in the tensile capacity and failure mode of the plates, thus a relatively large value, e.g., 100e2MPa in this case, was assigned to it to avoid any premature shear failure of the material. Therefore, the parameters to be calibrated are reduced to the three normal strengths. The calibration process is described in Section 3.3.

Fig. 150 compares the load-deformation curves obtained from the tests and the DEM modelling for the plates with and without holes. The above calibrated contact stiffness were used as the bond stiffness of the particles in the DEM models. It can been seen that the stiffness of plates calculated from the DEM models match well with the stiffness of plates from the tests. 


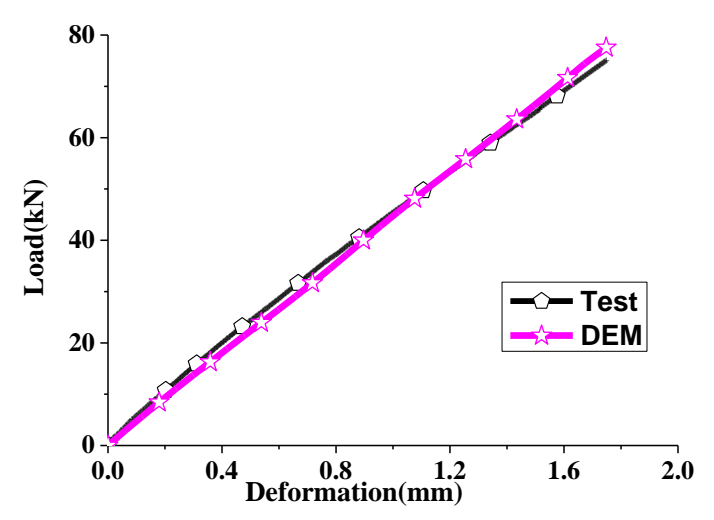

(a) plates without holes

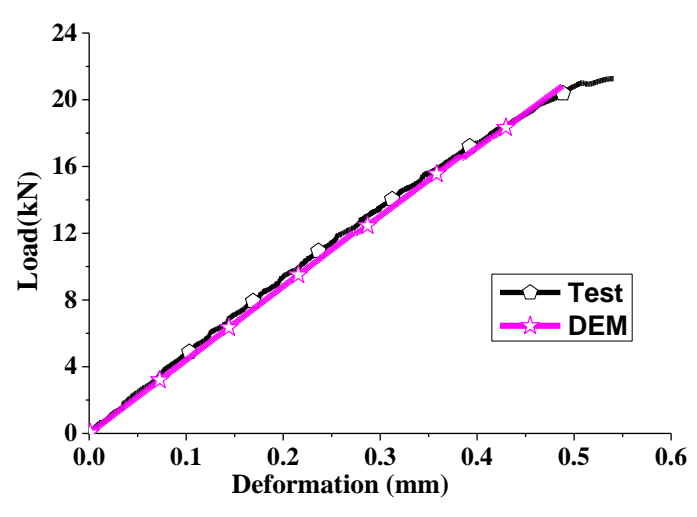

(b) plates with holes

Fig. 10 Load-deformation curves of the plates with and without holes

\subsection{Strength in the $x x$ and yy directions in the $x-y$ plane}

Under tensile loading, tensile strength in the $x x$ direction are represented by $\bar{\sigma}_{n x x}$ and $\bar{\sigma}_{n x y}$. The relative values of the two strengths offer three possible breaking sequences in the $\mathrm{xx}$ and the $x y$ directions, as shown in Fig. 1(a-c). These include (a) when $\bar{\sigma}_{n x x}$ and $\bar{\sigma}_{n x y}$ are proportional to each other such that the two contacts break simultaneously; (b) when $\bar{\sigma}_{n x x}$ is sufficiently smaller such that the $x x$ contact breaks first; and (c) when $\bar{\sigma}_{n x y}$ is sufficiently smaller such that the $x y$ contact breaks first. Through extensive simulations, it was found that the three breaking sequences were mainly related to the three stress-strain relations shown in Fig. 102(a-c). When the two contacts break at the same time, the stress increases linearly to the breaking point, then drops abruptly to zero (Fig. 102a). In this case, the values and proportionality of $\bar{\sigma}_{n x x}$ and $\bar{\sigma}_{n x y}$ can be calibrated by comparing with the test results. When the $x x$ contact breaks first, the stress reaches its limit specified by $\bar{\sigma}_{n x x}$ followed by an abrupt drop and a 'small strain hardening' to a second peak related to $\bar{\sigma}_{n x y}$ (Fig.12b). The second peak is normally lower then the first one if $\bar{\sigma}_{n x y}$ is not excessively high. When the $x y$ contact break first, there is a small drop in stress during the loading stage before reaching the final breaking point (Fig. 12c), where $\bar{\sigma}_{n x y}$ has a great influence on the failure mode and the peak value. In Fig. 102, the yellow and green zones denote the contributions from $\bar{\sigma}_{n x x}$ and $\bar{\sigma}_{n x y}$, respectively.

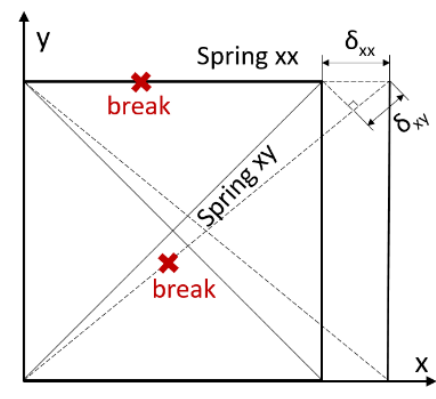

(a) break at the same time

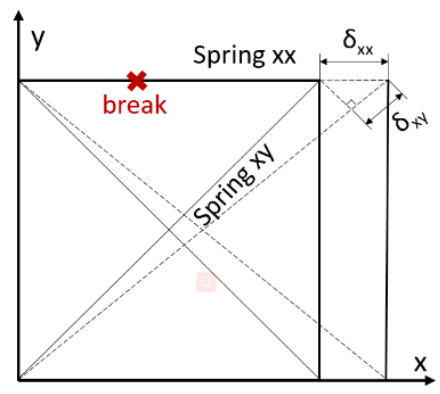

(b) contact $x x$ breaks firstly

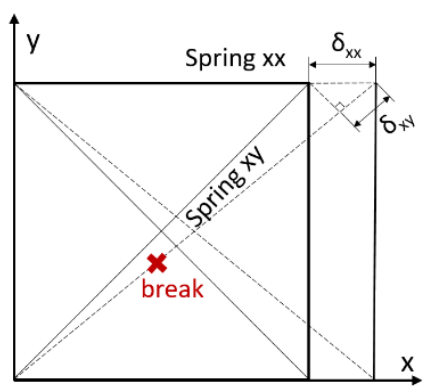

(c) contact $x y$ breaks firstly

Fig. 11 Three situations of contacts breaking of $x x$ and $x y$ 


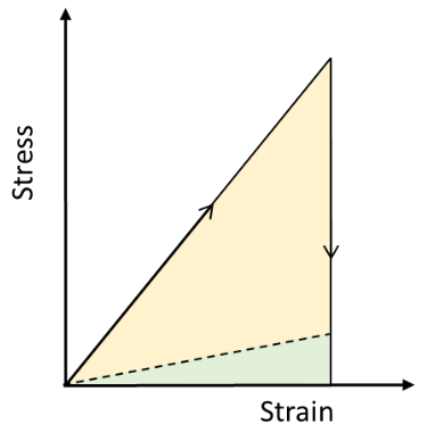

(a) break at the same time

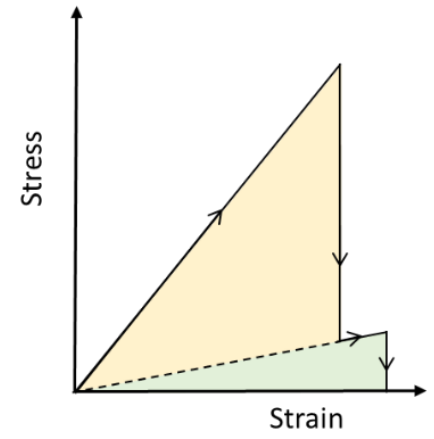

(b) spring $x x$ breaks firstly

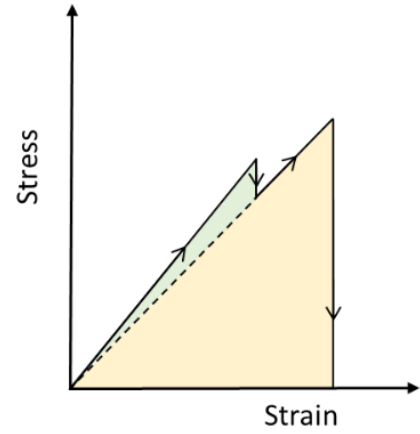

(c) spring $x y$ breaks firstly

Fig. 102 Strain-stress curves of the three breaking sequences

From the laboratory tests, there were no noticeable drops during the entire loading process. On the basis of the above discussion, it is, therefore, assumed that the $x x$ contact breaks earlier than the $x y$ contact does. Thus, a large value is assigned to $\bar{\sigma}_{n x y}$ (1000MPa in this paper) to ensure that it will not break before the $x x$ contact. $\bar{\sigma}_{n x x}$ is calibrated by changing its value to match the longitudinal strength $\sigma_{x}$ (the first peak value) of the plates from the tests, as shown in Fig. 113(a). It shows that $\sigma_{x}$ increases linearly with $\bar{\sigma}_{n x x}$. Thus, when $\bar{\sigma}_{n x x}$ is 330Mpa, $\sigma_{x}$ reaches $303.9 \mathrm{Mpa}$ which is close to both the strength of the plate tested in this paper and by Turvey and Szulik. By following a similar procedure, Fig. 113(b) can be plotted to show the correlation between the transverse strength $\sigma_{y}$ and $\bar{\sigma}_{n y y}$ of the plate, where when $\bar{\sigma}_{n y y}$ is about $55 \mathrm{MPa}, \sigma_{y}$ is close to the test result, i.e., $95.8 \mathrm{MPa}$.

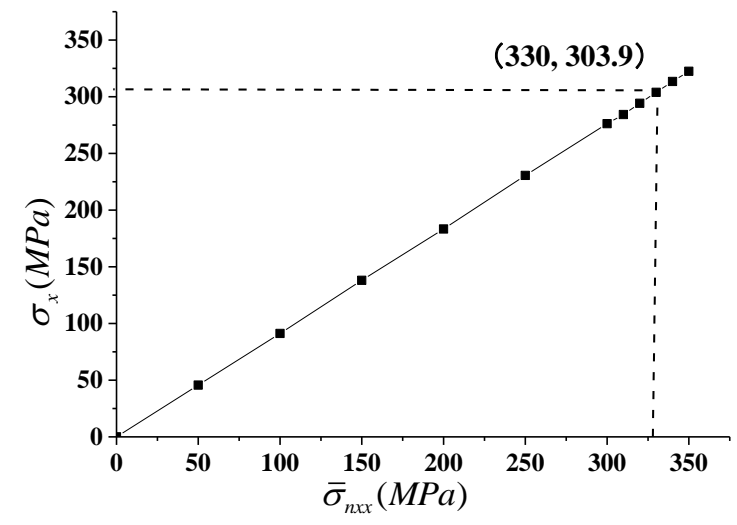

(a) $\bar{\sigma}_{n x x}$ changes

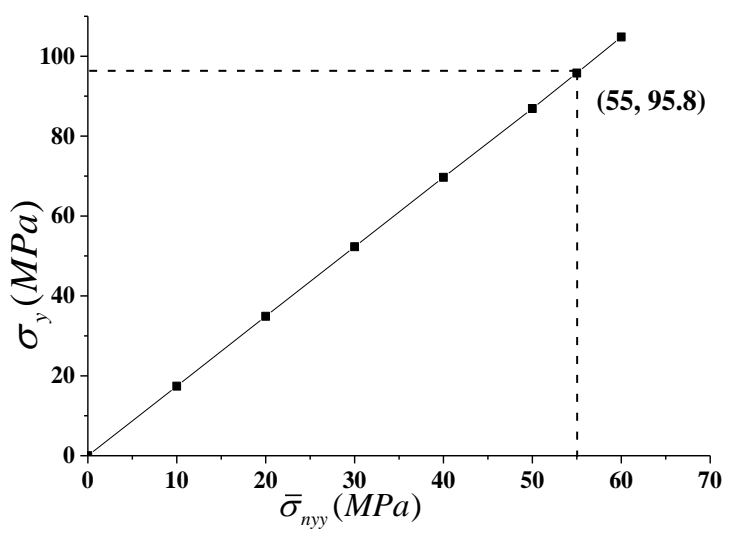

(b) $\bar{\sigma}_{n y y}$ changes

Fig. 113 Calibration of contact strength against the strength of the plates

\subsection{Strength of the $x y$ contact}

For plates with a hole, stress concentration exists around the hole during the loading process. Six evenly contacts numbered (1) to (6), as shown in Fig. 124(a), are chosen in the calibrations. On the cross section across the hole, the longitudinal stress is maximum at the boundary of the hole and the minimum at the transverse edge of the plate, as shown in Fig. 124 (b). The $x y$ contacts along the boundary of the hole is subjected to the highest stress and are prone to breaking first, resulting in progressive failure along in the transverse direction of the plate due to the brittleness of the material. Hence the strength in the $x y$ contact plays a significant role in resisting tension of the plates with holes. There are two possible failure modes in this model, one of which is that the $x y$ contact at the boundary of the hole on the smallest cross section of the plate fails first, and the other is that the 
$x x$ contact at a similar position breaks first, as shown in Fig. 135 (a) and (b), respectively, where the blue and the green denote, respectively, the contacts that are not damaged and have failed in shear. Fig. 14 shows the influence of $\bar{\sigma}_{n x y}$ on the failure load. When $\bar{\sigma}_{n x y}$ is smaller than 50Mpa, the $x y$ contact breaks first and $\bar{\sigma}_{n x y}$ has a significant influence on the tension capacity $F_{\max }$. When $\bar{\sigma}_{n x y}$ is larger than $50 \mathrm{MPa}$, the $x x$ contact breaks first, and the curve goes down and then becomes plateaued, suggesting that $F_{\max }$ is mainly determined by $\bar{\sigma}_{n x x}$ at this stage. From the calibration results, when $\bar{\sigma}_{n x y}=50 \mathrm{MPa}$, the failure load is close to the test values of $20.8 \mathrm{kN}$ (Fig.16). Table 3 summarizes the strength of the micro contacts within the $\mathrm{x}-\mathrm{y}$ plane from the above calibrations.

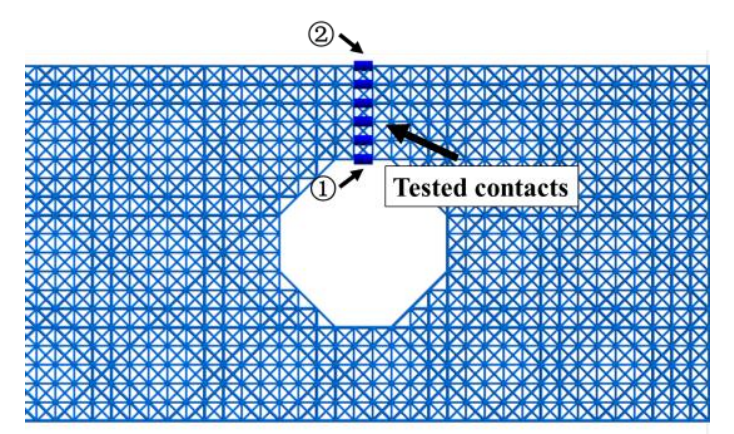

(a) tested contacts

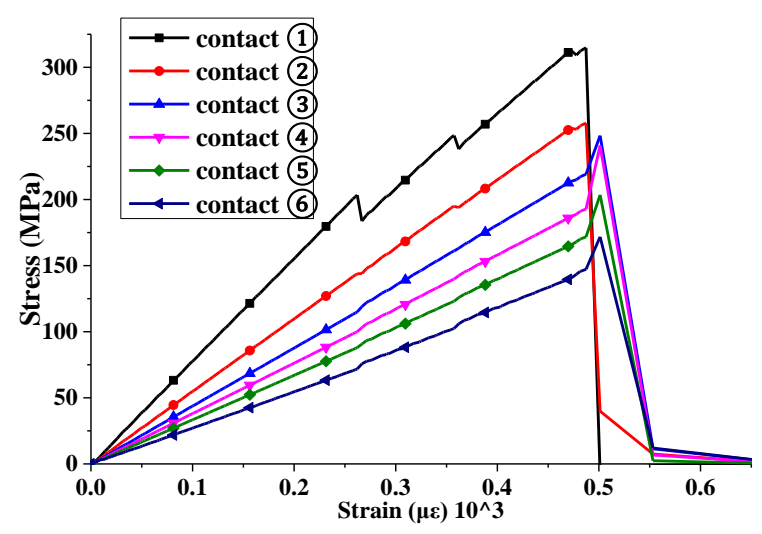

(b) Stress- strain curves of tested contacts

Fig. 124 Stress distribution around hole

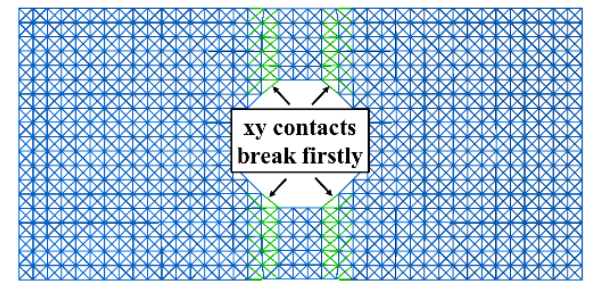

(a) $x y$ contacts break firstly

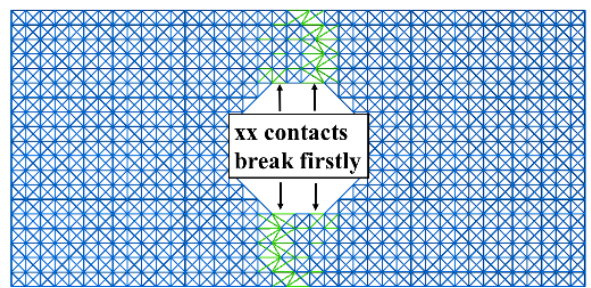

(b) $x x$ contacts break firstly

Fig. 135 Two possible failure modes

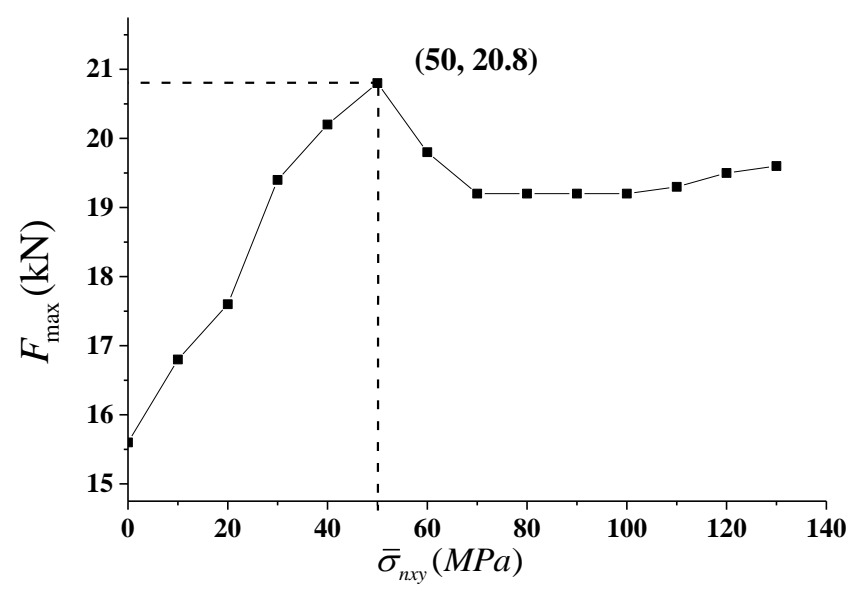

Fig. 146 Calibration of contact strength against the capacity of the plates

Table 3 Calibrated contact strength in $x-y$ plane

\begin{tabular}{llll}
\hline \multirow{2}{*}{ Calibrated strength } & $\bar{\sigma}_{n x x}$ & $\bar{\sigma}_{n y y}$ & $\bar{\sigma}_{n x y}$ \\
\cline { 2 - 4 } & $330 \mathrm{MPa}$ & $55 \mathrm{MPa}$ & $50 \mathrm{MPa}$ \\
\hline
\end{tabular}

Table 4 presents the predicted strength of the laminates using the DEM model with the contact properties shown in Tables 2 and 3 and compare them with the test results. It can be found that the predictions of the DEM model agree well with the experiment results for both laminates. The maximum error is within $4 \%$. It indicates 
that the calibrated stiffness and strength are suitable for simulating plates made of the GFRP. For a different material, e.g., Carbon-based FRP or Basalt-based FRP, the contact strength should be re-calibrated by following

Table 4 Comparison of DEM model against experiment results for plates with and without holes

\begin{tabular}{lllll}
\hline & & Experiments & DEM & Error \\
\hline \multirow{2}{*}{ Plates without holes } & Longitudinal strength & $293.0 \mathrm{MPa}$ & $303.9 \mathrm{MPa}$ & $3.6 \%$ \\
\cline { 2 - 5 } & Transverse strength & $95.4 \mathrm{MPa}$ & $95.8 \mathrm{MPa}$ & $0.42 \%$ \\
\hline Plates with holes & Tension capacity & $21.6 \mathrm{kN}$ & $20.8 \mathrm{kN}$ & $-3.7 \%$ \\
\hline
\end{tabular}

\section{DEM model for GFRP bolted joints}

\subsection{Tensile test of GFRP bolted joints}

The calibrations presented in Section 3 enable good agreement between the modelling and the laboratory tests to be achieved for the composite plates with and without holes. The failure of joints, however, is more complicated and significant deformation in the thickness direction, such as delamination and bending occurs. In order to study the mechanic behavior of GFRP bolted joints and further validate the DEM model proposed in the previous sections, laboratory tests were conducted on two groups of bolted joints of $25 \mathrm{~mm}$ and $40 \mathrm{~mm}$ lap length, respectively. Each group has three identical specimens, the geometry of which is shown in Fig.17. The bolts are manually tightened with a small tightness of $3 \mathrm{Nm}$ applied to ensure integrity. The low bolt torque is used to quantify the lower bound load capacity of the joint. As expected, an appropriately higher bolt torque can increase the strength of the joint. The tests were carried out following the same procedure as the tests of the plates and the deformation of the lapping area was measured.
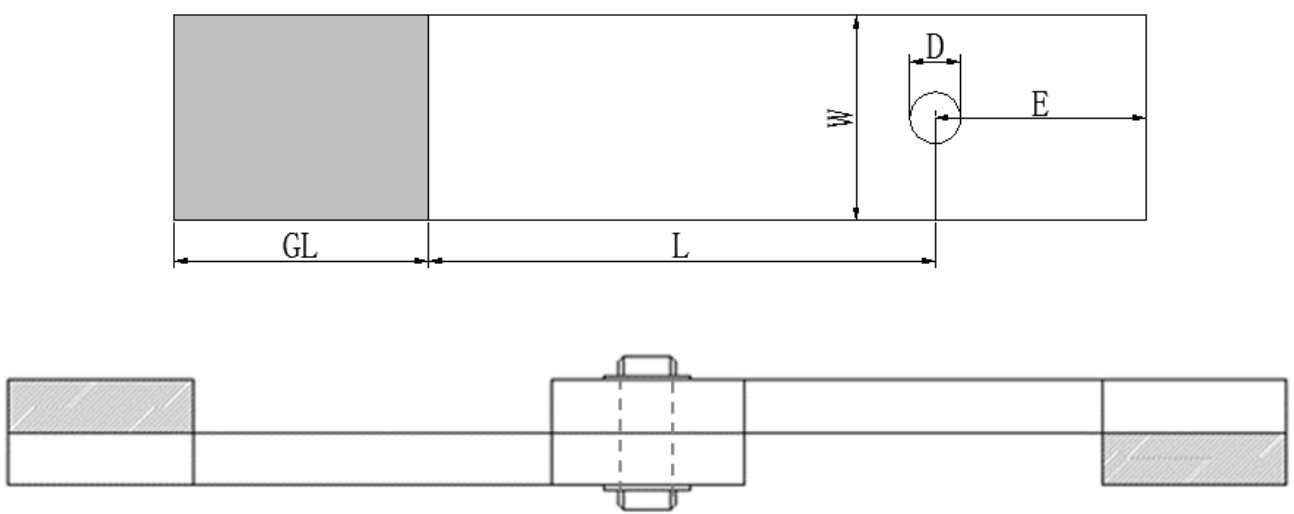

Length: $\mathrm{L}=100 \mathrm{~mm} \quad$ Specimen width: $\mathrm{W}=40 \mathrm{~mm}$

Hole diameter: $\mathrm{D}=10 \mathrm{~mm} \quad$ Grip length: $\mathrm{GL}=50 \mathrm{~mm}$
Specimen thickness: $\mathrm{H}=6.4 \mathrm{~mm}$

Overlap length: $\mathrm{E}=25 \mathrm{~mm} / 40 \mathrm{~mm}$

Fig. 15 Geometry of bolted joints

The test results showed that the lap length had impact on the failure mode of bolted joints. The mode for the joint with $25 \mathrm{~mm}$ lap length showed clear cleavage failure at the near end of the bolted region, as shown in 
Fig. 18 (a). It failed mainly due to brittle cracking initiated from the boundary of the hole and perpendicular to the edge of the plate. For the joint with lap length of $40 \mathrm{~mm}$, delamination in plates was the main reason for the failure. Transverse fracture occurred near the bolt, and progressive delamination developed with visible rotation caused by the eccentric tensile forces acting on the plate ends (Fig. 18b). The load verves deformation curves in Fig. 17 show that the three specimens of each lap length have similar slopes in the elastic stage. Comparing the failure of the joints of the two groups, the joints with $25 \mathrm{~mm}$ lap length fails brittlely while the joints with $40 \mathrm{~mm}$ lap length show obvious ductility. The average tensile capacities of the joints with lap length of $25 \mathrm{~mm}$ and $40 \mathrm{~mm}$ are $16.8 \mathrm{kN}$ and $17.1 \mathrm{kN}$, respectively.

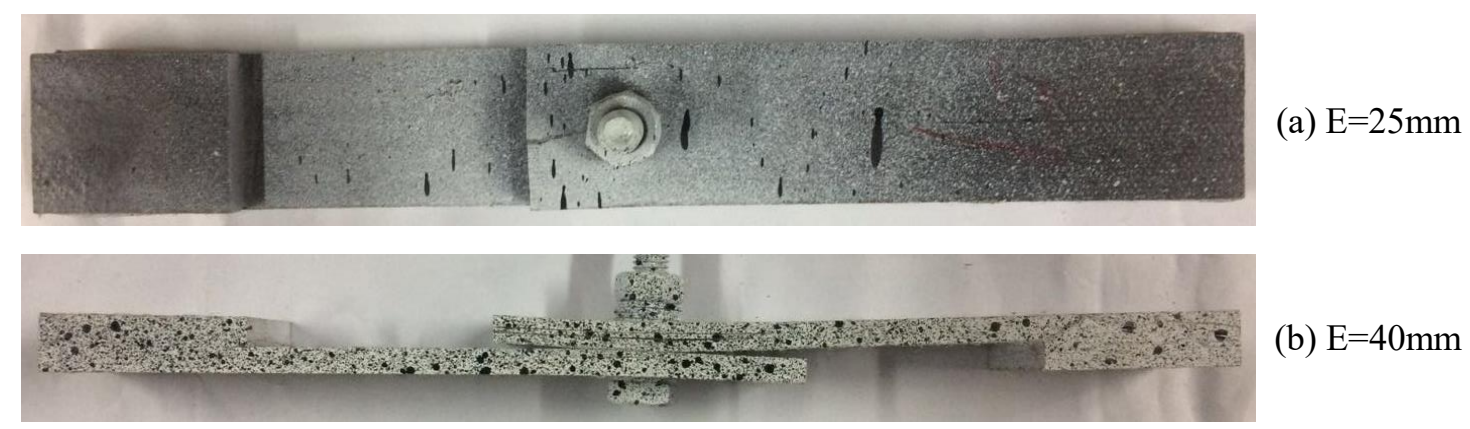

Fig. 16 Failure modes of bolted joints in test

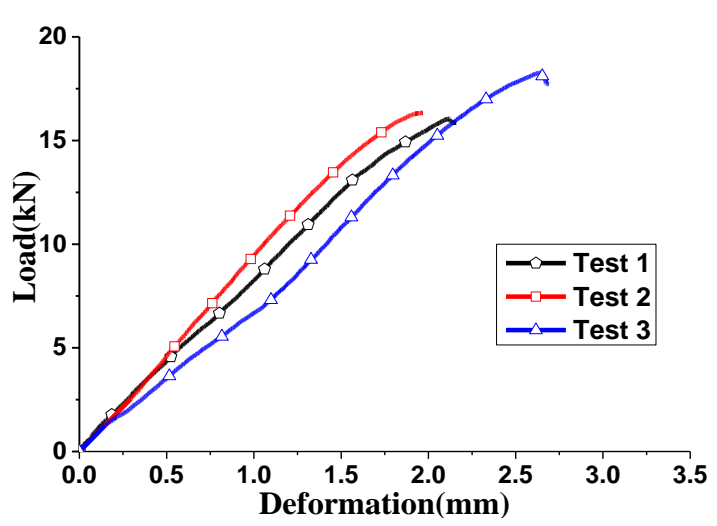

(a) $\mathrm{E}=25 \mathrm{~mm}$

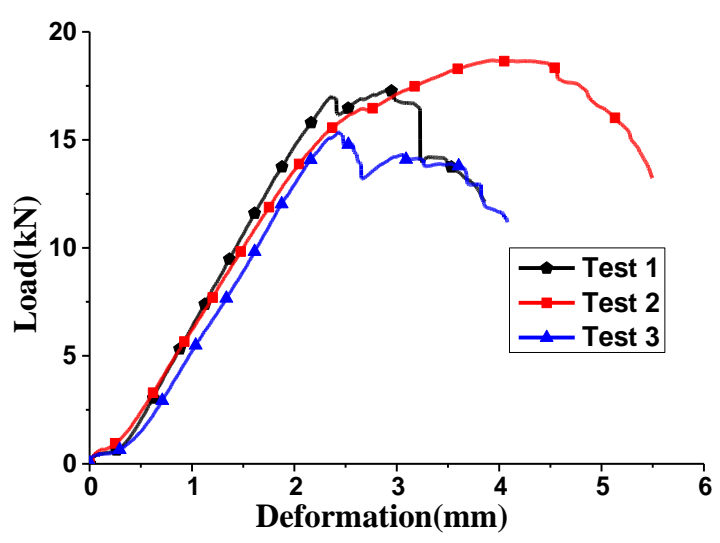

(b) $\mathrm{E}=40 \mathrm{~mm}$

Fig. 17 Load-deformation curves

\subsection{Calibration for strength of the zz contact}

Since bending occurs when the joint is subjected to tension, significant peeling and shearing take place on the interfaces of the laminates. The contact strength of the interfaces can now be calibrated using the test results, which cannot be done when using the previous plate models under pure tension.

The full scale of the DEM models of the tested joints with the two lap lengths are shown in as shown in Fig. 18. The bolt is modeled by a rigid clump of $10 \mathrm{~mm}$ diameter and the nuts at the two ends of the bolt restrict the out-of-plane displacement of the particles around the hole. Since the tightness of the bolts is small in the tests, the effect of the pre-stress in the $\mathrm{z}$ direction is ignored in this paper in order to reduce the complexity of the model. The contacts between the two plates and between the bolt and the particles of plates around the surface of the hole are frictional with a recommended friction coefficient of 0.5 . 


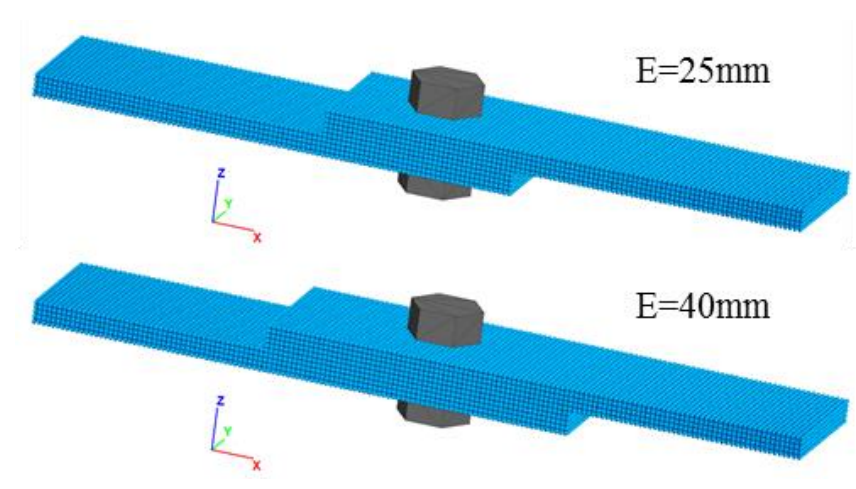

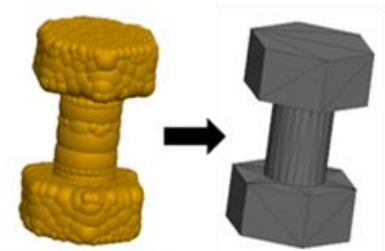

Clump for bolt

Fig. 18 DME model of bolted joints

To simulate the failure mode of bolted joints numerically, the normal and tangential strengths of the interfaces between the 5 layers play an important role. Because the bolted joint with $\mathrm{E}=25 \mathrm{~mm}$ mainly failed of in-plane cleavage due to the tension in the $y y$ direction and the tangential strength $\bar{\sigma}_{s z z}$ mainly controls interlaminar shear strength of plates, it is reasonable to assume that $\bar{\sigma}_{s z z}$ is sufficiently big to maintain integrity. $\bar{\sigma}_{n z z}$ is changed the to simulate the $\mathrm{E}=25 \mathrm{~mm}$ joint to achieve the target load capacity and the observed failure mode, as shown in Fig. 19. When $\bar{\sigma}_{n z z}$ increases to $60 \mathrm{MPa}$, the tension capacity reaches $16.4 \mathrm{kN}$ and maintains almost constantly afterwards. By taken this value for the interfacial normal strength, the predicted failure modes are also comparable to the observations from the experimental tests.

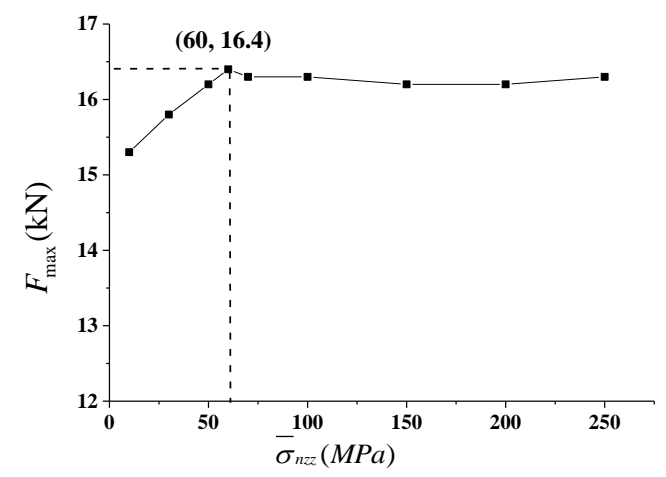

Fig. 19 Calibration of interfacial normal strength

For the 40mm lap length joint, the tests shown clear delamination that are largely attributed to the shear strength, $\bar{\sigma}_{s z z}$, of the interface. The interfacial shear strength is calibrated here aiming at achieving both comparable load capacity with the test results and comparable failure mode, including delamination. The parametric evaluations are presented in Fig. 20Error! Reference source not found.. There are two loading stages of the curve. At the first stage, the load capacity can be improved rapidly by increasing $\bar{\sigma}_{s z z}$, which indicates that $\bar{\sigma}_{s z z}$ plays a significant role in preventing delamination at this stage. At the second stage, $\bar{\sigma}_{s z z}$ 's contribution in increasing load capacity becomes insignificant and damage has occurred due to other loss of strength. After comparing both the load capacity and the failure modes observed from the experiments. $\bar{\sigma}_{s z z}$ $=100 \mathrm{MPa}$ is chosen as the interfacial shear strength of the laminates. 


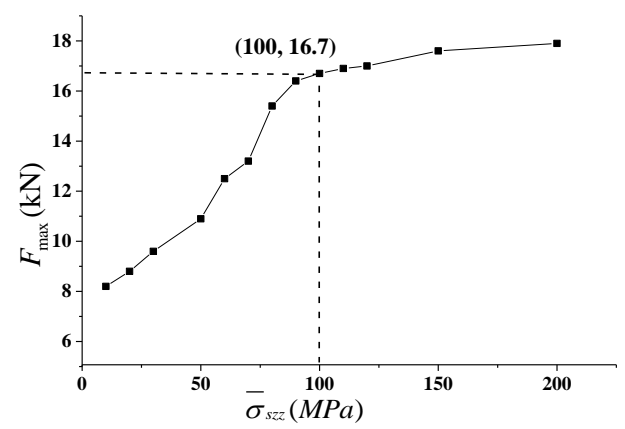

Fig. 20 Calibration of interfacial normal strength

\subsection{Discrete element model for bolted joints}

Based on calibrated strength above, two joint model are established and validation and analysis are discussed in this section. The numerical results for the $E=25$ joint are shown in Fig. 21Error! Reference source not found. and are compared with the experimental tests. Fig. 21 (a) is the full scale model of the joint showing the final failure mode. Fig. 21 Error! Reference source not found.(b) presents the comparisons between the load-displacement from the model and the test results, which shows very good agreement. The predicted maximum load from the model is $16.4 \mathrm{kN}$, representing a $2.4 \%$ error in comparison with the test result of $16.8 \mathrm{kN}$. The close-up view of the failure of the test specimen is shown in Fig. 21Error! Reference source not found.(c). The joint fails by brittle cleavage, which is also captured by the DEM model inError! Reference source not found. Fig. 21 (d). The simulation also show some micro/small cracks around the hole, which are hardly observed on the specimen by the naked eyes. Fig. 21 (f) shows the side view of the DEM model illustrating rotation of the joint, which agrees well with the test shown in Fig. 21 (e). There is noticeable rotation, while no visible delamination.

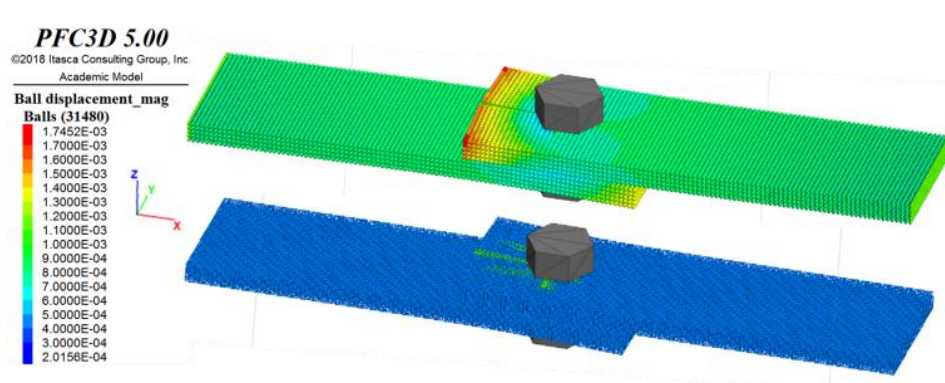

(a) entire model for $\mathrm{E}=25$ bolted joint

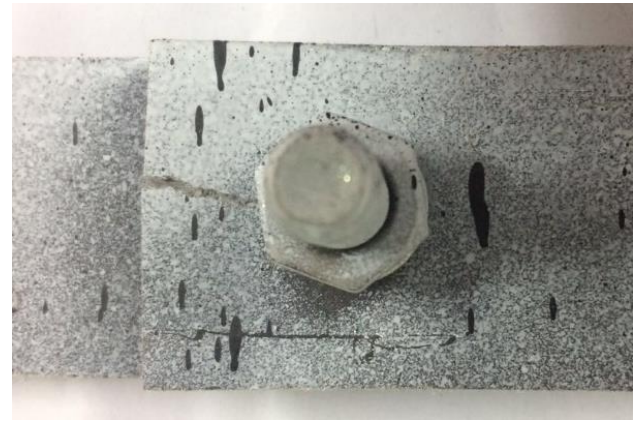

(c) cracking in test

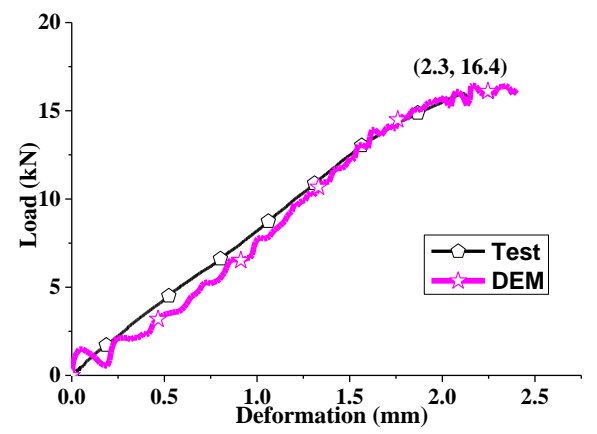

(b) load-deformation curve

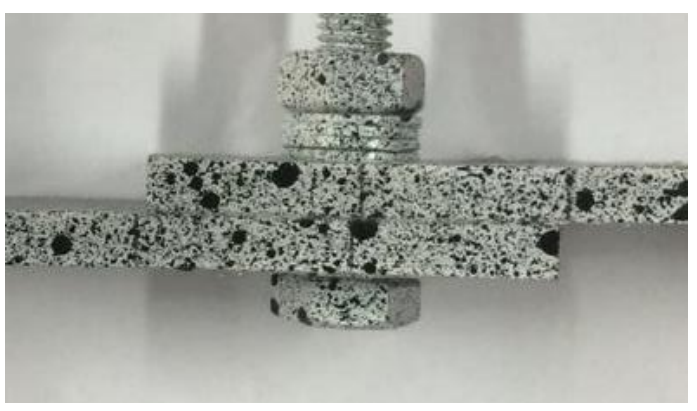

(e) rotation in test 

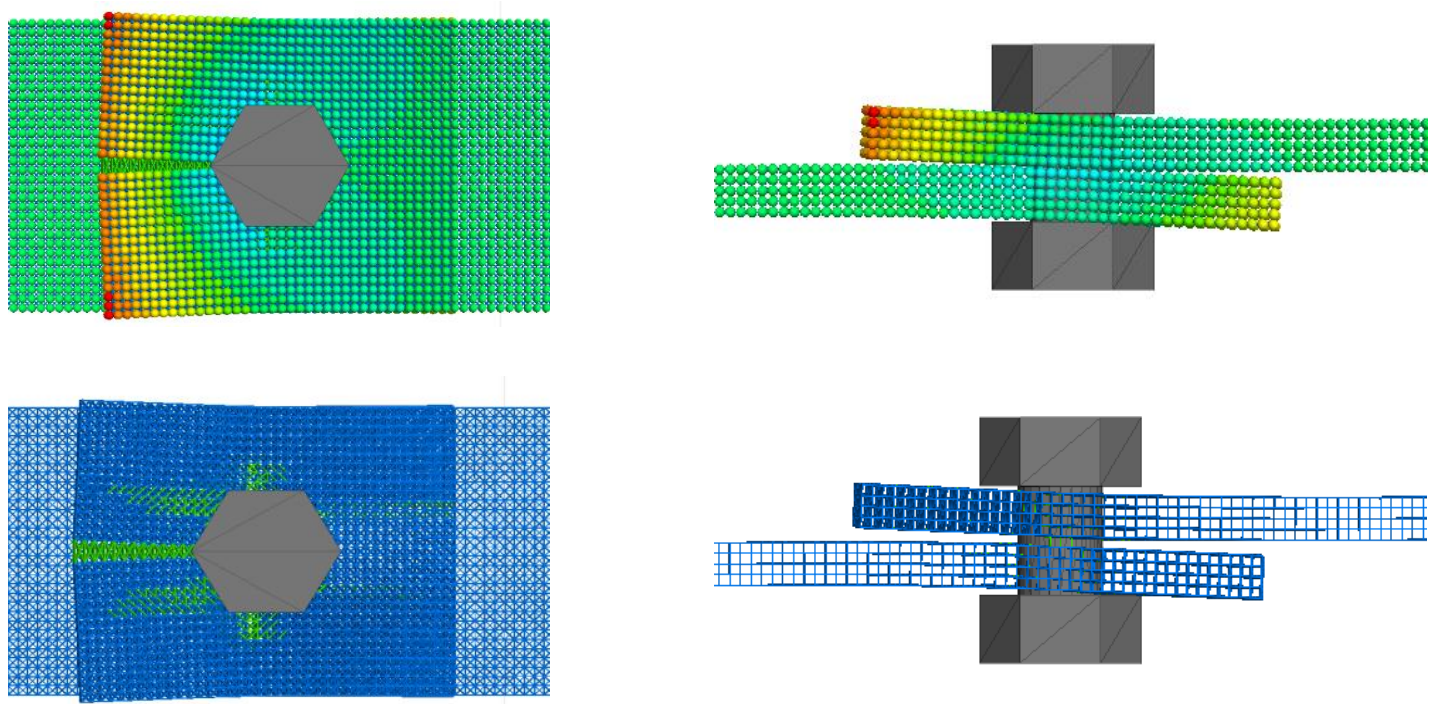

(d) cracking in DEM model

(f) rotation in DME model

Fig. 21 Modelling of the $25 \mathrm{~mm}$ lap length joints

Fig. 22Error! Reference source not found. shows the failure for the $E=40$ bolted joint. Because delamination was observed from the experiments, various side views of the model are discussed. From Fig. 22(a)Error! Reference source not found., the model does not show any cracks on the top surface, while visible damage are shown by the larger displacements of the lighter colored particles concentrated at the center of the up-plate. The DEM model also shows plastic deformation of the joint due to the longer lap length, as shown in Fig. 22Error! Reference source not found.(b). The predicted tension capacity from the model is $16.7 \mathrm{kN}$, representing only $2.3 \%$ error in comparison with the test value of $17.1 \mathrm{kN}$. Fig. 22 (c) and (d) show comparable rotational and bending deformation of joint. The sectional view of the joint in Fig. 22 (e-f) reveals the internal damage due to delamination. The three cuts of Fig. 22 (e) are taken along the length direction at $y=0.01 \mathrm{~m}, 0.02$ and $0.03 \mathrm{~m}$, respectively. The three cuts in Fig. 22 (f) are taken transversely at $\mathrm{x}=0.100 \mathrm{~m}, 0.106 \mathrm{~m}$ and $0.112 \mathrm{~m}$. In the figures, the red $z z$ contacts denote that the interfacial shear strength has been exceeded and deamination has occurred. It is found that longest interfacial delamination occurs at the middle longitudinal section, as shown in the Section 2 of Fig. 22Error! Reference source not found.(e). The three transvers sections (Sections 4-6) show that the some cracks propagate to one side of the bolt and some to the other side, which is consistent with the crack formation observed in the tests.

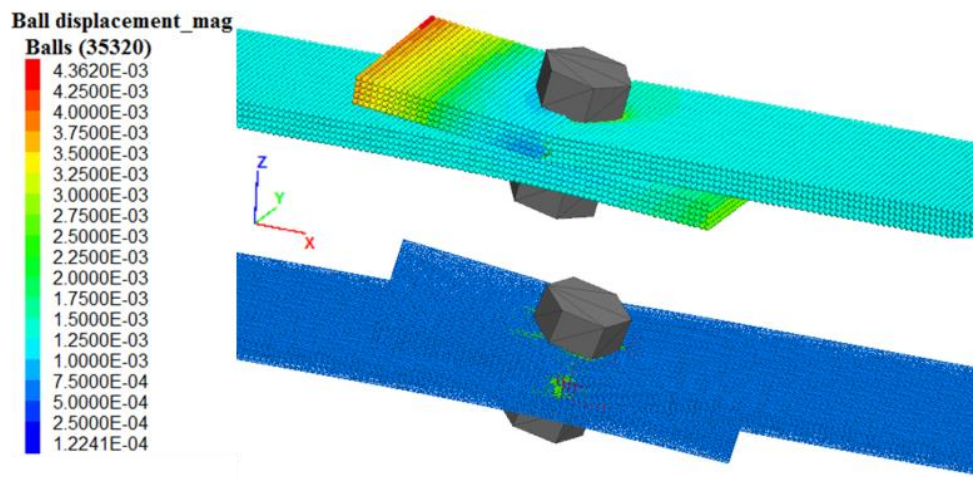

(a) entire model for $\mathrm{E}=40$ bolted joint

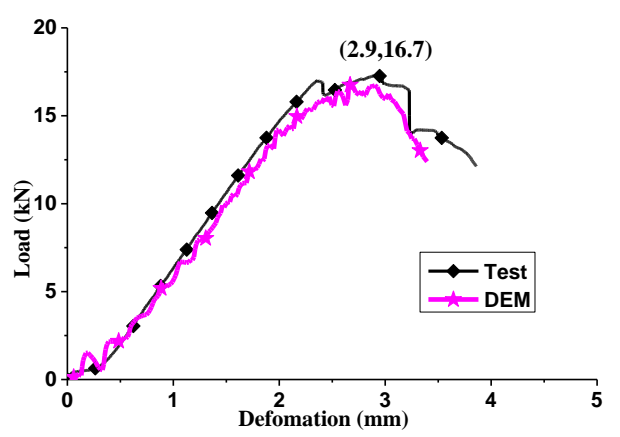

(b) load-deformation curve 


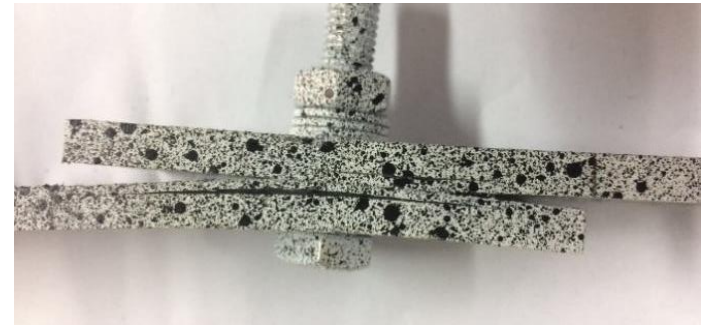

(c) delamination and bending in test

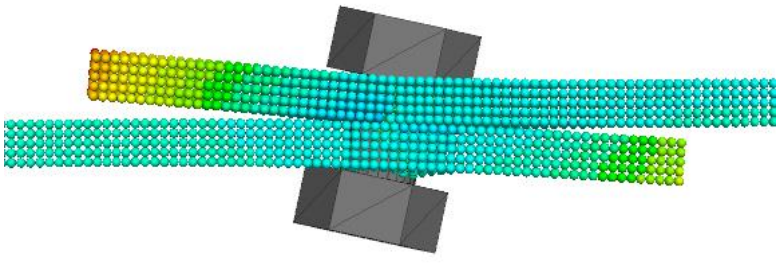

(d) delamination in DEM model

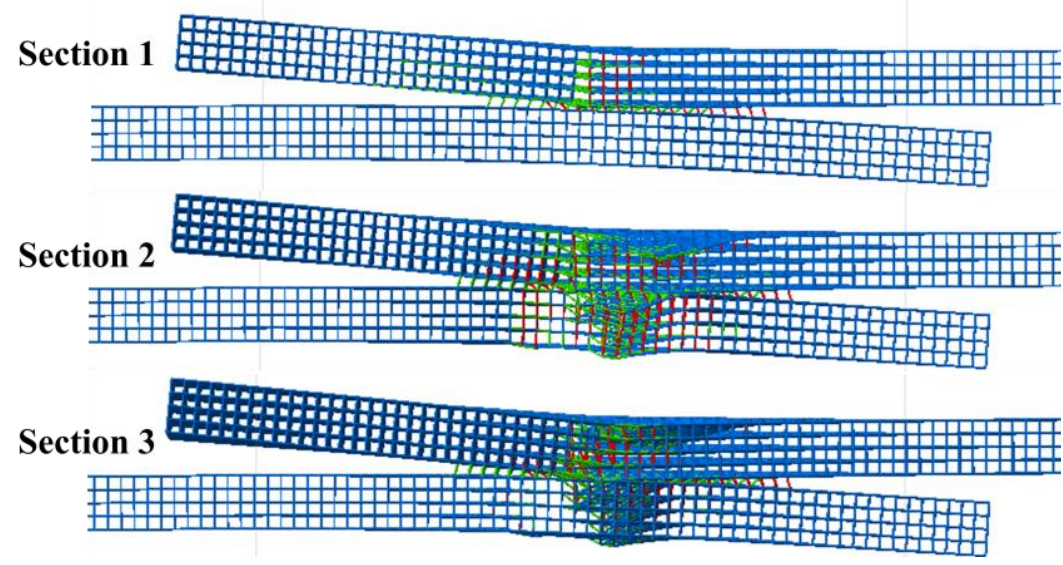

(e) longitudinal sections

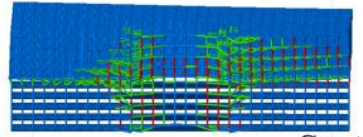

Section 4



(f) transverse sections

Fig. 22 Modelling of the $40 \mathrm{~mm}$ lap length joints

\section{Conclusions}

In this study, a new 19-ball 3D DEM model has been developed to model general orthotropic materials. Explicit relationships between the macro elastic constants and the micro contact stiffness were established. The model was the reduced to transversely isotropic, so that it could be used for modelling pultruded GFRP with homogenized material properties. The derived stiffness formulas are applicable to any other orthotropic or transversely isotropic materials

In order to celibate the micro contact properties of the DEM model, uniform thin plates, thin plate with a central hole and bolted joints were tested experimentally to obtain strength of the micro contacts between particles. A well defined calibration process has been developed. The procedure can applied to laminates made of other composites. However, improved calibration process for the contact properties of more complex materials requires further investigations.

To the authors' best knowledge, the work reported in this paper is the first attempt to simulate bolted joints by DEM and demonstrate the potential of the method. Obviously, to apply the method robustly to larger scale and more complex structures, significant future research is required. Inevitably, computation cost is one of the concerns, though currently a high performance computer are capable of handling billions of particles, optimization of the algorithms through parallel or/and GPU computing can significantly reduce the cost. Combination of DEM with other numerical approaches is obviously another option, by which only the parts that require micro/meso scale analysis are modelled by DEM, while other parts are simulated by, e.g., FEM.

It can be concluded that form the work presented in this paper DEM is a powerful alternative to other numerical methods, especially when details analysis of material damage are required, though future research is still required. 


\section{Acknowledgement}

The authors are grateful for the financial support from the National Key Research and Development Program (2017YFC0703001).

\section{References}

[1] S Guanlin, H Gengkai, L Bin. Mechanics of Composite Materials(Second Edition)[M]. Beijing: Tsinghua University Press, 2006.(in Chinese)

[2] Monaldo E, Nerilli F, Vairo G. Basalt-Based Fiber-Reinforced Materials and Structural Applications in Civil Engineering[J]. Composite Structures, 2019,214:246-263.

[3] Keller T. Recent All-Composite and Hybrid Fibre-Reinforced Polymer Bridges and Buildings[J]. Progress in Structural Engineering \& Materials, 2001,3(2):132-140.

[4] Monaldo E, Nerilli F, Vairo G. Effectiveness of some Technical Standards for Debonding Analysis in FRPConcrete Systems[J]. Composites Part B: Engineering, 2019,160:254-267.

[5] Hull D, Clyne T W. An Introduction to Composite Materials[J]. Concise Encyclopedia of Composite Materials, 2018,2(1):xvii-xxix.

[6] Chambers R E. Plastics Composites for 21St Century Construction[J]. New York NY American Society of Civil Engineers, 1993.

[7] Bhatnagar N, Ramakrishnan N, Naik N K, et al. On the Machining of Fiber Reinforced Plastic (FRP) Composite Laminates[J]. International Journal of Machine Tools \& Manufacture, 1995,35(5):701-716.

[8] Sayman O, Siyahkoc R, Sen F, et al. Experimental Determination of Bearing Strength in Fiber Reinforced Laminated Composite Bolted Joints Under Preload[J]. Journal of Reinforced Plastics and Composites, 2007,26(10):1051-1063.

[9] Turvey G J. Bolted Joints in Pultruded Glass Fibre Reinforced Polymer (GFRP) Composites[J]. Composite Joints \& Connections, 2011:77-111.

[10] Ireman T, Ranvik T, Eriksson I. On Damage Development in Mechanically Fastened Composite Laminates[J]. Composite Structures, 2000,49(2):151-171.

[11] Pinho S T, Darvizeh R, Robinson P, et al. Material and Structural Response of Polymer-Matrix FibreReinforced Composites[J]. Journal of Composite Materials, 2012,46(19-20):2313-2341.

[12] Lu Z, Xian G, Li H. Experimental Study On the Mechanical Properties of Basalt Fibres and Pultruded BFRP Plates at Elevated Temperatures[J]. Polymers and Polymer Composites, 2015,23(5):277-284. 
[13] Kandare E, Kandola B K, Myler P, et al. Thermo-Mechanical Responses of Fiber-Reinforced Epoxy Composites Exposed to High Temperature Environments. Part I: Experimental Data Acquisition[J]. Journal of Composite Materials, 2010,44(26):3093-3114.

[14] Cao S, Wu Z, Wang X. Tensile Properties of CFRP and Hybrid FRP Composites at Elevated Temperatures[J]. Journal of Composite Materials, 2009,43(4):315-330.

[15] Hinton M. Failure Criteria in Fibre-Reinforced-Polymer Composites[M]. 2004.

[16] Hinton M J, Kaddour A S. Special Issue: Evaluation of Theories for Predicting Failure in Polymer Composite Laminates Under 3-D States of Stress: Part A of the Second World-Wide Failure Exercise (WWFE-II) Foreword[J]. Journal of Composite Materials, 2012,46(19-20SI):2281-2282.

[17] Kaddour A S, Hinton M J, Smith P A, et al. The Background to the Third World-Wide Failure Exercise[J]. Journal of Composite Materials, 2013,47(20-21):2417-2426.

[18] Kaddour A S, Hinton M J. Maturity of 3D Failure Criteria for Fibre-Reinforced Composites: Comparison Between Theories and Experiments: Part B of WWFE-II [J]. Journal of Composite Materials, 2013,47(67SI):925-966.

[19] Hinton M J, Kaddour A S. The Background to Part B of the Second World-Wide Failure Exercise: Evaluation of Theories for Predicting Failure in Polymer Composite Laminates Under Three-Dimensional States of Stress[J]. Journal of Composite Materials, 2013,47(6-7SI):641.

[20] Kaddour A S, Hinton M J, Smith P A, et al. Mechanical Properties and Details of Composite Laminates for the Test Cases Used in the Third World-Wide Failure Exercise[J]. Journal of Composite Materials, 2013,47(20-21):2427-2442.

[21] Hosokawa Y, Katayama T, Nakayama M, et al. Life Prediction Under Sulfuric Acid Environment of FRP Using X-Ray Analysis Microscope[J]. Journal of Materials Processing Technology, 2004,155-156:15581563.

[22] S. G T, L. B W. In-Situ Observations in SEM of Degradation of Graphite/Epoxy Composite Materials Due to Seawater Immersion[J]. Journal of Composite Materials, 1995,29(7):852-867.

[23] Mccarthy M A, Mccarthy C T. Finite Element Analysis of Effects of Clearance On Single Shear Composite Bolted Joints[J]. Plastics, Rubber and Composites, 2013,32(2):65-70.

[24] Sheng H Y, Ye J Q. A State Space Finite Element for Laminated Composite Plates[J]. Computer Methods in Applied Mechanics and Engineering, 2002,191(PII S0045-7825(02)00379-137-38):4259-4276.

[25] Turvey G J, Wang P. An Fe Analysis of the Stresses in Pultruded Grp Single-Bolt Tension Joints and their Implications for Joint Design[J]. Computers \& Structures, 2008,86(9):1014-1021.

[26] Mccarthy M A, Mccarthy C T, Lawlor V P, et al. Three-Dimensional Finite Element Analysis of SingleBolt, Single-Lap Composite Bolted Joints: Part I-Model Development and Validation[J]. Composite Structures, 2005,71(2):140-158.

[27] Nerilli F, Vairo G. Progressive Damage in Composite Bolted Joints Via a Computational Micromechanical Approach[J]. Composites Part B: Engineering, 2017,111:357-371. 
[28] Citarella R, Cricri G. Three-Dimensional BEM and FEM Submodelling in a Cracked FML Full Scale Aeronautic Panel[J]. Applied Composite Materials, 2014,21(3):557-577.

[29] Freddi F, Salvadori A, Savoia M. Boundary Element Analysis of FRP-Concrete Delamination.[J]. Boundary Elements XXVI, 2004:335-344.

[30] Kaminski M. Stochastic Boundary Element Method Analysis of the Interface Defects in Composite Materials[J]. Composite Structures, 2012,94(2):394-402.

[31] Ismail Y, Yang D, Ye J. A DEM Model for Visualising Damage Evolution and Predicting Failure Envelope of Composite Laminae Under Biaxial Loads[J]. Composites Part B: Engineering, 2016,102:9-28.

[32] Cundall P A, Strack O D L. Discussion: A Discrete Numerical Model for Granular Assemblies[J]. Géotechnique, 1980,30(3):331-336.

[33] Cundall P A, Strack O D L. A Discrete Numerical Model for Granular Assemblies[J]. Geotechnique, 2008,29(30):331-336.

[34] Zha X, Wan C, Fan Y, et al. Discrete Element Modeling of Metal Skinned Sandwich Composite Panel Subjected to Uniform Load[J]. Computational Materials Science, 2013,69:73-80.

[35] Yang D, Sheng Y, Ye J, et al. Discrete Element Modeling of the Microbond Test of Fiber Reinforced Composite[J]. Computational Materials Science, 2010,49(2):253-259.

[36] Wan L, Yang D, Ismail Y, et al. 3D Particle Models for Composite Laminates with Anisotropic Elasticity[J]. Composites Part B: Engineering, 2018,149:110-121.

[37] Sheng Y, Yang D, Tan Y, et al. Microstructure Effects On Transverse Cracking in Composite Laminae by DEM[J]. Composites Science and Technology, 2010,70(14):2093-2101.

[38] Ismail Y, Sheng Y, Yang D, et al. Discrete Element Modelling of Unidirectional Fibre-Reinforced Polymers Under Transverse Tension[J]. Composites Part B-Engineering, 2015,73:118-125.

[39] Yang D, Ye J, Tan Y, et al. Modeling Progressive Delamination of Laminated Composites by Discrete Element Method[J]. Computational Materials Science, 2011,50(3):858-864.

[40] Yang D, Sheng Y, Ye J, et al. Dynamic Simulation of Crack Initiation and Propagation in Cross-Ply Laminates by DEM[J]. Composites Science and Technology, 2011,71(11):1410-1418.

[41] Maheo L, Dau F, André D, et al. A Promising Way to Model Cracks in Composite Using Discrete Element Method[J]. Composites Part B: Engineering, 2015,71:193-202.

[42] Le B D, Dau F, Charles J L, et al. Modeling Damages and Cracks Growth in Composite with a 3D Discrete Element Method[J]. Composites Part B: Engineering, 2016,91:615-630.

[43] Safranyik F, Keppler I, Bablena A. DEM Calibration: A Complex Optimization Problem[C], 2017.

[44] Group I C. Pfc3D Version 5.0[CP/OL].

[45] Tavarez F A, Plesha M E. Discrete Element Method for Modelling Solid and Particulate Materials[J]. International Journal for Numerical Methods in Engineering, 2007,70(4):379-404.

[46] Liu K, Liu W. Application of Discrete Element Method for Continuum Dynamic Problems[J]. Archive of 
Applied Mechanics, 2006,76(3-4):229-243.

[47] Liu C, Xu Q, Shi B, et al. Mechanical Properties and Energy Conversion of 3D Close-Packed Lattice Model for Brittle Rocks[J]. Computers \& Geosciences, 2017,103:12-20.

[48] Zhao G, Fang J, Zhao J. A 3D Distinct Lattice Spring Model for Elasticity and Dynamic Failure[J]. International Journal for Numerical and Analytical Methods in Geomechanics, 2011,35(8):859-885.

[49] Turvey G J, Szulik M. Thermo-Mechanically Loaded Glass-Fibre-Reinforced Polymer Single-Bolt SingleLap Joints[J]. Structures \& Buildings, 2017,171(11):1-12. 